\title{
Efficient Exact Schedulability Tests for Fixed Priority Real-time Systems
}

\author{
Robert Davis, Attila Zabos, and Alan Burns \\ Real-Time Systems Research Group, Department of Computer Science, \\ University of York, YO10 5DD, York (UK) \\ rob.davis@cs.york.ac.uk, attila@cs.york.ac.uk, alan.burns@cs.york.ac.uk
}

\begin{abstract}
Efficient exact schedulability tests are required both for on-line admission of applications to dynamic systems and as an integral part of design tools for complex distributed real-time systems. This paper addresses performance issues with exact Response Time Analysis (RTA) for fixed priority pre-emptive systems. Initial values are introduced that improve the efficiency of the standard RTA algorithm (i) when exact response times are required, and (ii) when only exact schedulability need be determined. The paper also explores modifications to the standard RTA algorithm, including; the use of a response time upper bound to determine when exact analysis is needed, incremental computation aimed at faster convergence, and checking tasks in reverse priority order to identify unschedulable tasksets early. The various initial values and algorithm implementations are compared by means of experiments on a PC recording the number of iterations required, and execution time measurements on a real-time embedded microprocessor. Recommendations are provided for engineers tasked with the problem of implementing exact schedulability tests, as part of on-line acceptance tests and spare capacity allocation algorithms, or as part of off-line system design tools.
\end{abstract}

\section{Introduction}

Fixed priority pre-emptive scheduling is widely used in real-time embedded systems, and is supported by the majority of commercial real-time operating systems.

In the context of fixed priority pre-emptive systems, schedulability analysis is used to determine if a set of tasks can be guaranteed to always meet their deadlines at run-time.

A schedulability test is referred to as sufficient if all tasksets deemed to be schedulable by the test are in fact schedulable. Similarly, a schedulability test is referred to as necessary if all tasksets deemed to be unschedulable by the test are in fact unschedulable. Schedulability tests that are both sufficient and necessary are referred to as exact.

In this paper, we are concerned with exact schedulability tests for fixed priority pre-emptive systems. Although these tests are known to be pseudo-polynomial in complexity [5], [6], [11], the scale of many commercial systems is such that exact tests can be used.

Efficient, exact schedulability tests are required for:

1. Admission of applications to dynamic systems at run-time.

2. Design of complex real-time systems, where schedulability analysis forms part of some higher level process of system optimisation.

Reducing the execution times of exact schedulability tests is an important consideration in these practical applications.

Two distinct approaches to constructing exact schedulability tests have appeared in the literature. These may be summarised as follows:

1. Response Time Analysis (RTA) [5], [11]. These approaches compute a worst-case response time for each task, which can then be checked against its deadline to determine schedulability.

2. Workload based tests [6], [19]. These approaches compute the task workload released prior to defined points in time, corresponding to the release times of higher priority tasks. The test then determines task schedulability by checking if the processor can complete the workload by each of the defined points.

In the appendix, we show that exact schedulability tests based on Response Time Analysis [11] are in general more computationally efficient than the most effective Workload based tests [19]. The remainder of this paper is therefore concerned with improving the performance of exact schedulability tests based upon Response Time Analysis.

We can classify the requirements for exact schedulability tests as follows:

1. Boolean schedulability tests: only a Boolean answer, either schedulable or not schedulable is required.

2. Response time tests: in the case of a schedulable system, the exact worst-case response time of each task is required. 
For on-line admission tests, a Boolean schedulability test is often sufficient; however, for use off-line, as part of a system design tool, response time tests are typically required. For example, in a distributed system based on Controller Area Network (CAN), the response times of tasks that read sensor data and then output information on the CAN bus affect the release jitter of messages sent on the bus [25]. Knowing exact worst-case response times for the tasks makes possible accurate analysis of message worst-case response times, and hence derivation of exact end-to-end response times from input event to output response.

Response Time Analysis can be used to provide both exact response time tests and exact Boolean schedulability tests. The latter more efficiently, as we will see in Sections 4 and 6.

\subsection{Motivation}

The motivation for this research comes from the Frescor project [23]. The Frescor scheduling framework supports the execution of multiple applications on a single processor. Each application is executed within its associated periodic server which has a capacity, period, and deadline. The servers run under a fixed priority pre-emptive scheduler. Determining server schedulability is effectively an analogous problem to computing the schedulability of a set of periodic/sporadic tasks.

Applications can be added to a Frescor system at runtime. Before a new application can be added, the admission test needs to check that all the existing servers remain schedulable, and that the additional server supporting the new application is also schedulable.

Once an application has been admitted to the system, the scheduling framework must determine the amount of spare capacity to allocate to each of those applications requesting additional capacity. The spare capacity allocation algorithm makes multiple calls to the schedulability test to determine the feasibility of the system with respect to different allocations of spare capacity.

Whilst it is envisaged that some Frescor based systems will be large and complex, it is expected that the total number of applications running on a single processor will not be excessive (e.g. less than 100). To achieve the best possible performance in terms of the applications that can be admitted, and the spare capacity that can be allocated, it is therefore desirable to use an exact schedulability test. As the schedulability test must be carried out on-line, and completed before a new application can start, it is important that the schedulability test is as efficient as possible. This desire to provide an efficient and effective schedulability test for use in the Frescor scheduling framework motivates our research.

As the research presented in this paper is applicable to the widely used fixed priority pre-emptive tasking model, in the remainder of the paper, we will use the term task to mean the schedulable entity of interest, which in the case of Frescor may, in fact, be a server or virtual resource.

\subsection{Related research}

Research into schedulability tests for fixed priority preemptive systems effectively began in 1967, when Fineberg and Serlin [1] considered priority assignment for two tasks. They noted that if the task with the shorter period is assigned the higher priority, then the least upper bound on the schedulable utilisation is $2(\sqrt{2}-1)$ or $82.8 \%$. This result was generalised by both Serlin [2] in 1972 and Liu and Layland [3] in 1973, both of whom showed that for synchronous tasks (that share a common release time), that comply with a restrictive system model, and that have deadlines equal to their periods $\left(D_{i}=T_{i}\right)$, then rate monotonic ${ }^{1}$ priority ordering (RMPO) is optimal. Liu and Layland [3] provided the following sufficient schedulability test for tasks compliant with their model, and with priorities assigned according to RMPO:

$\sum_{i=1 . . . n} U_{i} \leq n\left(2^{1 / n}-1\right)$

Where $U_{i}=C_{i} / T_{i}$ is the utilisation of task $\tau_{i}$, and $n$ is the number of tasks.

In 1982, Leung and Whitehead showed that deadline monotonic ${ }^{2}$ priority ordering (DMPO) [4] is optimal for tasks with deadlines less than or equal to their periods $\left(D_{i} \leq T_{i}\right)$.

Subsequently, exact schedulability tests were developed by Joseph and Pandya [5] in 1986, Lehoczky et al. [6] in 1989, and Audsley et al. [11] in 1993. These exact tests have been extended to cater for cases where tasks access mutually exclusive shared resources according to mechanisms such as the Stack Resource Policy [9] developed by Baker from the Priority Ceiling Protocol (PCP) of Sha et al. [7].

Further work on schedulability tests for fixed priority systems has lifted many of the earlier restrictions, providing exact tests for tasks with offset release times [10], arbitrary deadlines $\left(D_{i}>T_{i}\right)$ [8],[12], and non-preemptive sections [21]; these extensions are however beyond the scope of this paper.

Improvements to the performance of exact response time tests effectively began with Audsley [22] in 1993, who provided an initial value, for use in the recurrence relation used to compute task worst-case response times, that was based on the response time of the next higher priority task.

In 1998 Sjodin and Hansson [13] extended Audsley's work, by accounting for blocking factors in the initial value calculation. They also introduced a closed form lower bound on the response time that could be used as an effective initial value. Sjodin and Hansson showed that these initial values lead to fewer iterations of the recur-

\footnotetext{
${ }^{1} \mathrm{RMPO}$ assigns priorities in order of task periods, such that the task with shortest period is given the highest priority.

${ }^{2}$ DMPO assigns priorities in order of task deadlines, such that the task with the shortest deadline is given the highest priority.
} 
rence relation and quantified the improvements in algorithm performance.

In 2003, Bril et al. [14] considered online response time calculations using similar initial values to those introduced by Audsley, and Sjodin and Hansson.

The initial values used by Audsley [22], Sjodin and Hansson [13], and Bril et al. [14] are all lower bounds on the worst-case response time, thus exact worst-case response time can be found starting from these values.

In 2007, $\mathrm{Lu}$ et al. [17] introduced two new "deadline dependent" initial values, which can be used to determine exact schedulability, but cannot in general be used to find exact worst-case response times. Lu et al. showed that significant efficiency gains are possible using these new initial values combined with previous ones.

Previous research by Lu et al. in 2006 [16] sought to improve the performance of response time analysis by partitioning higher priority tasks into two sets. Interference from one set of tasks was then treated as consuming execution time according to their utilisation, leaving a fraction of the processor available for computation due to the remaining tasks. This approach reduced the number of iterations of the algorithm required for convergence.

Related work by Bini and Buttazzo introduced the Hyperplanes Exact Test (HET) [19] in 2004. The Hyperplanes Exact Test provides a means of improving the efficiency of the exact schedulability test formulated by Lehoczky et al. in [6], via a reduction in the number of points in time at which the workload needs to be evaluated.

The research presented in this paper builds upon the work of Sjodin and Hansson [13], and Bril et al. [14]. It takes the concept of task partitioning introduced by $\mathrm{Lu}$ et al. [16] and uses it to form a new series of initial values that can be used in exact response time tests. The research also builds upon the work of $\mathrm{Lu}$ et al. [17] providing two improved initial values for use in exact Boolean schedulability tests.

\subsection{Organisation}

Section 2 gives the system model, terminology and notation used in the rest of the paper, along with a recapitulation of the standard Response Time Analysis (RTA) recurrence relation. Section 3 introduces a new series of initial values, the largest of which can be used to compute exact worst-case response times. Section 4 presents two improved initial values that can be used in Boolean schedulability tests. Section 5 discusses improvements to the schedulability test algorithm, including an incremental approach, the use of a response time upper bound ensuring that exact schedulability computation is only performed when necessary, and reversing the order in which task schedulability is checked with the aim of identifying unschedulable tasks more quickly. Section 6 outlines an empirical investigation into schedulability test efficiency. This is complemented by Section 7 which provides execu- tion time measurements from an implementation of the schedulability tests on an embedded microprocessor. Section 8 gives our recommendations to engineers tasked with implementing exact schedulability tests. Section 9 concludes with a summary of the main contributions of the paper and an outline of areas for future research. Finally, additional material in the appendix provides a comparison between schedulability tests, based on Response Time Analysis, discussed in this paper, and the Hyperplanes Exact Test (HET) described in [19], showing that the latter is not as effective as previously thought.

\section{System model and basic analysis}

\subsection{Terminology and notation}

In this paper, we are interested in providing efficient, exact schedulability tests for applications executing under a fixed priority pre-emptive scheduler on a single processor. The application is assumed to comprise a static set of $n$ tasks $\left(\tau_{1} . . \tau_{n}\right)$, each assigned a unique priority $i$, from 1 to $n$ (where $n$ is the lowest priority).

We use the notation $h p(i)$ and $l p(i)$ to mean the set of tasks with priorities higher than $i$, and the set of tasks with priorities lower than $i$ respectively. Similarly, we use the notation hep(i) and lep(i) to mean the set of tasks with priorities higher than or equal to $i$, and lower than or equal to $i$ respectively.

Application tasks may arrive either periodically at fixed intervals of time, or sporadically after some minimum inter-arrival time has elapsed. Each task $\tau_{i}$, is characterised by: its relative deadline $D_{i}$, worst-case execution time $C_{i}$, minimum inter-arrival time or period $T_{i}$, and release jitter $J_{i}$, defined as the maximum time between the task arriving and it being released (ready to execute). It is assumed that once a task starts to execute it will never voluntarily suspend itself.

Tasks may make mutually exclusive access to shared resources according to the Stack Resource Policy (SRP) [9]. A task at priority $i$ may be blocked by lower priority tasks, as a result of the operation of the SRP, for at most $B_{i}$, referred to as the blocking time.

A task's worst-case response time $R_{i}$, is the longest time from the task becoming ready to execute to it completing execution. A task is referred to as schedulable if its worst-case response time is less than or equal to its deadline less release jitter $\left(R_{i} \leq D_{i}-J_{i}\right)$. A system is referred to as schedulable if all its tasks are schedulable.

We assume that task deadlines are less than or equal to their periods $D_{i} \leq T_{i}$, and without loss of generality that task priorities are in deadline minus jitter monotonic ${ }^{3}$ (D-JMPO) priority order [20].

\footnotetext{
${ }^{3}$ D-JMPO assigns priorities in order of deadline minus jitter, such that the task with the smallest value of $D_{i}-J_{i}$ is given the highest priority.
} 


\subsection{Busy periods and idle instants}

The concept of a busy period, introduced by Lehoczky in [8], is fundamental in analysing worst-case response times. The following concepts are used in the analysis presented in subsequent sections.

A priority level-i idle instant is defined as a time instant $t$ at which there are no tasks of priority $i$ or higher awaiting execution that became ready to execute strictly before time $t$.

A priority level-i busy period is defined as follows:

1. It starts at a priority level-i idle instant $t^{s}$, when a task of priority $i$ or higher becomes ready to execute.

2. It is a contiguous interval of time during which any task of priority lower than $i$ is unable to begin executing.

3. It ends at the first priority level-i idle instant $t^{e}$, following $t^{s}$.

A critical instant [3], for task $\tau_{i}$, is defined as a time at which task $\tau_{i}$ becomes ready to execute, and is then subject to the maximum possible delay, i.e. its worst-case response time, before completing execution. For tasks complying with the system model outlined above, a critical instant occurs when task $\tau_{i}$ becomes ready to execute simultaneously with all tasks of higher priority, and subsequent invocations of these higher priority tasks become ready as soon as possible. Further, immediately before task $\tau_{i}$ is released, a lower priority tasks locks a resource with a ceiling priority of $i$ or higher, resulting in the maximum blocking time $B_{i}$. For this system model, the worst-case response time of task $\tau_{i}$ is equivalent to the length of the longest priority level-i busy period.

\subsection{Basic response time analysis}

Response time analysis calculates the length of the longest priority level-i busy period and hence the worst-case response time of task $\tau_{i}$, using the following equation.

$R_{i}=B_{i}+C_{i}+\sum_{\forall j \in h p(i)}\left\lceil\frac{R_{i}+J_{j}}{T_{j}}\right\rceil C_{j}$

Note that the worst-case response time $R_{i}$ appears on both the left and right hand side of Equation (2). As the right hand side is a monotonically non-decreasing function of $R_{i}$, the equation can be solved using the following recurrence relation:

$r_{i}^{n+1}=B_{i}+C_{i}+\sum_{\forall j \in h p(i)}\left\lceil\frac{r_{i}^{n}+J_{j}}{T_{j}}\right\rceil C_{j}$

Iteration starts with an initial value $r_{i}^{0}$, typically $r_{i}^{0}=B_{i}+C_{i}$, and ends when either $r_{i}^{n+1}=r_{i}^{n}$ in which case the worst-case response time $R_{i}$, is given by $r_{i}^{n+1}$ or when $r_{i}^{n+1}>D_{i}-J_{i}$ in which case the task is unschedulable.

In general, Equation (2) may have a number of different solutions, the smallest of which corresponds to the worst-case response time $R_{i}$. The recurrence relation is guaranteed to converge on the smallest solution $R_{i}$, if and only if the initial value $r_{i}^{0}$ is less than or equal to $R_{i}$, thus any initial value less than or equal to $R_{i}$ will suffice to determine the exact value of $R_{i}$.

Further, for any two initial values $r^{A}$ and $r^{B}$ where $r^{A} \leq r^{B} \leq R_{i}$, then the number of iterations $N^{A}$, of the recurrence relation required to converge on the solution $R_{i}$ from initial value $r^{A}$ is at least as great as the number of iterations $N^{B}$, required when starting from initial value $r^{B}$. Stated otherwise, the largest possible initial value $\leq R_{i}$ will result in the least number of iterations and the hence the fastest possible convergence.

\subsection{Performance metrics}

A number of different metrics could be used to explore the performance of the recurrence relation:

1. Number of iterations of the recurrence relation required for convergence.

2. Total number of ceiling operations required for convergence.

3. Execution time of a specific implementation on a particular microprocessor.

In their experiments, Sjodin and Hansson [13], and Lu et al. [17] used the number of iterations of the recurrence relation as a performance metric. By contrast, Bril et al. [14] used the number of ceiling operations. We argue that the latter is a better metric, as each iteration requires a variable number of ceiling operations dependent on the priority of the task. Thus using iterations as a measure could potential skew the results, if for example, a particular approach required less iterations for high priority tasks, but more for those of low priority.

In our empirical investigations, in Section 6, we use the number of ceiling operations as a performance metric, and as a simple proxy for the later execution time measurements made in Section 7.

\section{Initial values for exact response time tests}

In this section, we consider initial values for exact response time tests.

\subsection{Previous work}

In 1993, in chapter 4 of his thesis, Audsley [22] showed that, for systems of independent tasks, with task schedulability tested in priority order, $R_{i-1}+C_{i}$ is an effective initial value.

In 1998, Sjodin and Hansson [13] extended this lower bound on $R_{i}$ to account for blocking:

$R_{i}^{L B}=R_{i-1}-B_{i-1}+B_{i}+C_{i}$

By approximating the ceiling function in the recurrence relation by a division operation, Sjodin and Hansson [13] also introduced the following closed form lower bound: 


$$
R_{i}^{L B}=\frac{B_{i}+C_{i}+\sum_{\forall j \in h p(i)} J_{j} U_{j}}{1-\sum_{\forall j \in h p(i)} U_{j}}
$$

In [14], Bril et al. derived essentially the same lower bounds for a simple scheduling model, assuming no jitter or blocking:

$$
\begin{aligned}
& R_{i}^{L B}=R_{i-1}+C_{i} \\
& \quad \text { and } \\
& R_{i}^{L B}=\frac{C_{i}}{1-\sum_{\forall j \in h p(i)} U_{j}}
\end{aligned}
$$

We note that using the initial values given by Equations (4) and (6) requires that task response times are determined in priority order, highest priority first.

\subsection{New initial values}

We now introduce a series of new initial values, the maximum of which can be used to provide a later starting point, reducing the number of iterations required by the recurrenc erelation.

For each priority level $i$, there are $i$ initial values in the series. To form each new initial value, identified by the index $k(k=1 \ldots i)$, we partition the set of tasks of higher than or equal priority to $i$, i.e. $h e p(i)$, into two sets: $h p(k)$ and $\operatorname{lep}(k) \cap h e p(i)$.

Following the approach of $\mathrm{Lu}$ et al. in [16], we consider the tasks in $h p(k)$ as taking a proportion of the available processing time $\alpha_{k}$ where:

$$
\alpha_{k}=\sum_{\forall j \in h p(k)} U_{j}
$$

Thus only a fraction of the processing time $1-\alpha_{k}$ remains available to accommodate the remaining task load. Given that $R_{i} \geq R_{i-1}$, the contribution of each task in hep(i) to the total task load in $R_{i}$ is at least $I_{j}\left(R_{i-1}\right)$, where $I_{j}\left(R_{i-1}\right)$ is the worst-case interference due to task $\tau_{j} \in h p(i)$ occurring during the response time of task $\tau_{i-1}$.

$I_{j}\left(R_{i-1}\right)=\left\lceil\frac{R_{i-1}+J_{j}}{T_{j}}\right\rceil C_{j}$

We note that as the response time of task $\tau_{i}$ is only computed if task $\tau_{i-1}$ is schedulable, $I_{i-1}\left(R_{i-1}\right)=C_{i-1}$.

Using this information, we compose the series of $i$ lower bounds on $R_{i}$ corresponding to each priority $k$ from 1 to $i$..

$$
R_{i}^{L B}(k)=\frac{B_{i}+C_{i}+\sum_{\forall j \in l e p(k) \cap h p(i)} I_{j}\left(R_{i-1}\right)}{1-\sum_{\forall j \in h p(k)} U_{j}}
$$

The largest such bound is given by:

$$
R_{i}^{L B}=\max _{\forall k=1 . . i}\left(R_{i}^{L B}(k)\right)
$$

We note that this set of lower bounds has as its first and last members, the two initial values proposed in [13] and [14].

For $k=1$ :

$$
\begin{aligned}
R_{i}^{L B}(1)= & B_{i}+C_{i}+\sum_{\forall j \in h p(i)} I_{j}\left(R_{i-1}\right) \\
& =B_{i}+C_{i}+C_{i-1}+\sum_{\forall j \in h e p(i)} I_{j}\left(R_{i-1}\right) \\
& =B_{i}+C_{i}+R_{i-1}-B_{i-1}
\end{aligned}
$$

equivalent to Equations (4) and (6).

For $k=i$ :

$$
R_{i}^{L B}(i)=\frac{B_{i}+C_{i}}{1-\sum_{\forall j \in h p(k)} U_{j}}
$$

which, for systems with no release jitter, is equivalent to Equations (5) and (7).

We note that for tasksets with no release jitter, the new bound given by Equation (11) subsumes and therefore dominates the bounds given by Equations (4) to (7).

\subsection{Examples}

We now show using a simple example that the lower bound calculated via Equation (11) can be greater than the lower bounds computed via Equations (4) to (7). The example is based on the task parameters described in Table 1 below. The overall utilisation of this taskset is $92.5 \%$. The tasks are assumed to be independent, and have zero release jitter. Note, the final column in the table, headed $\mathrm{U}^{*}$, is the cumulative utilisation for all higher priority tasks.

TABLE 1: TASK PARAMETERS

\begin{tabular}{llllll}
\hline Priority & C & D & T & U & U* \\
\hline 1 & 5 & 10 & 10 & 0.5 & 0 \\
2 & 25 & 100 & 100 & 0.25 & 0.5 \\
3 & 25 & 200 & 200 & 0.125 & 0.75 \\
4 & 30 & 1000 & 1200 & 0.025 & 0.875 \\
5 & 30 & 1200 & 1200 & 0.025 & 0.9 \\
\hline
\end{tabular}

Our example considers the lower bounds (initial values) for the calculation of $R_{5}$. Assuming that response times are calculated in priority order, we have the following information available from the calculation of $R_{4}$ : $R_{4}=360$, comprising $I_{1}\left(R_{4}\right)=180, \quad I_{2}\left(R_{4}\right)=100$, $I_{3}\left(R_{4}\right)=50$, and $I_{4}\left(R_{4}\right)=C_{4}=30$.

The series of lower bounds $R_{5}^{L B}(k)$ are therefore:

$$
\begin{aligned}
& R_{5}^{L B}(0)=\left(I_{1}+I_{2}+I_{3}+I_{4}+C_{5}\right) / 1=390 \\
& R_{5}^{L B}(1)=\left(I_{2}+I_{3}+I_{4}+C_{5}\right) / 0.5=420 \\
& R_{5}^{L B}(2)=\left(I_{3}+I_{4}+C_{5}\right) / 0.25=440 \\
& R_{5}^{L B}(3)=\left(I_{4}+C_{5}\right) / 0.125=480 \\
& R_{5}^{L B}(4)=C_{5} / 0.1=300
\end{aligned}
$$

The largest such bound, $R_{5}^{L B}(3)$, is 480 . This is a significant improvement on the previous bounds of 390 and 300 given by Sjodin and Hansson [13], and Bril et al. 
[14]. In this example, $R_{5}=570$, which takes 7 iterations to calculate starting with an initial value of 480 , or 10 iterations starting with an initial value of 390 .

\section{Initial values for exact Boolean schedulability tests}

In this section, we consider initial values for exact Boolean schedulability tests.

\subsection{Previous work}

In 2007, Lu et al. [17] introduced two initial values $D_{i} / 2$ and $D_{i}-D_{i-1}$ that can be used as a starting point for the recurrence relation. However, unlike all of the initial values discussed in Section 3, these initial values are not necessarily lower bounds on $R_{i}$ and so do not guarantee that the recurrence relation will converge on the first solution (i.e. the exact worst-case response time). However, in their analysis $\mathrm{Lu}$ et al. showed that using these initial values the recurrence relation is guaranteed to converge on some upper bound $R_{i}^{U B}$, such that $R_{i} \leq R_{i}^{U B} \leq D_{i}$, if $\tau_{i}$ is in fact schedulable. Hence these initial values can be used to implement an exact Boolean schedulability test, but not an exact response time test.

\subsection{New initial values}

We now build upon the work of $\mathrm{Lu}$ et al. [17], extending their initial values so that they are applicable to the more general case of systems with blocking and release jitter. We then derive improved initial values that dominate those introduced by Lu et al.

Theorem 1. The initial value $\left(D_{i}-J_{i}\right)-\left(D_{i-1}-J_{i-1}\right)$ guarantees that the recurrence relation will converge to a solution $R_{i}^{U B}$, where $R_{i} \leq R_{i}^{U B} \leq D_{i}-J_{i}$ if $\tau_{i}$ is in fact schedulable.

Proof. We assume that task schedulability is determined in priority order and therefore that $\tau_{i-1}$ is schedulable. As $\tau_{i-1}$ is schedulable, there must be at least one priority level $i-1$ idle instant in any interval of length $R_{i-1}$ or greater (such as $D_{i-1}-J_{i-1}$ ).

To prove the theorem, there are two cases to consider:

1. $\tau_{i}$ is schedulable with $R_{i} \geq\left(D_{i}-J_{i}\right)-\left(D_{i-1}-J_{i-1}\right)$. In this case, the initial value $\left(D_{i}-J_{i}\right)-\left(D_{i-1}-J_{i-1}\right)$ is less than the first solution to the recurrence relation, and so the equation is guaranteed to converge on $R_{i}$.

2. $\tau_{i}$ is schedulable with $R_{i}<\left(D_{i}-J_{i}\right)-\left(D_{i-1}-J_{i-1}\right)$. In this case, as $\tau_{i}$ has completed execution by $\left(D_{i}-J_{i}\right)-\left(D_{i-1}-J_{i-1}\right)$, only tasks in $h p(i)$ are able to execute after this point. As the longest priority level-(i-1) busy period is known to be of length $R_{i-1} \leq D_{i-1}-J_{i-1}$, a further idle instant must occur by $\left(D_{i}-J_{i}\right)-\left(D_{i-1}-J_{i-1}\right)+R_{i-1}$ $\leq D_{i}-J_{i}$. The recurrence relation is therefore guaranteed to converge on some value $R_{i}^{U B}$, where $R_{i} \leq R_{i}^{U B} \leq D_{i}-J_{i}$

We now improve on the initial value given by Theorem 1 .

Theorem 2. The initial value $\left(D_{i}-J_{i}\right)-R_{i-1}^{U B}$, where $R_{i-1}^{U B}$ is an upper bound on the response time of $\tau_{i-1}$, and $\tau_{i-1}$ is known to be schedulable, so $R_{i-1}^{U B} \leq\left(D_{i-1}-J_{i-1}\right)$, guarantees that the recurrence relation will converge to a solution $R_{i}^{U B}$, where $R_{i} \leq R_{i}^{U B} \leq D_{i}-J_{i}$ if $\tau_{i}$ is in fact schedulable.

Proof. Follows directly from the proof of Theorem 1

As these methods do not guarantee to find exact response times, we use an upper bound $R_{i-1}^{U B}$ in Theorem 2. If an exact response time is known for $\tau_{i-1}$, then this provides the best possible upper bound.

Theorem 3. The starting value $\left(D_{i}-J_{i}\right) / 2$ guarantees that the recurrence relation will converge to a solution $R_{i}^{U B}$, where $R_{i} \leq R_{i}^{U B} \leq D_{i}-J_{i}$ if $\tau_{i}$ is in fact schedulable.

Proof. We first note the following two points:

1. Blocking occurs at the start of the busy period and so any delay to the execution of $\tau_{i}$ due to a lower priority task executing at priority $i$ or higher due to the operation of the Stack Resource Policy, must be greater in the interval $\left[0,\left(D_{i}-J_{i}\right) / 2\right)$ than it is in the interval $\left[\left(D_{i}-J_{i}\right) / 2,\left(D_{i}-J_{i}\right)\right)$.

2. Interference due to each task in $h p(i)$ must be greater in the interval $\left[0,\left(D_{i}-J_{i}\right) / 2\right)$ than it is in the interval $\left[\left(D_{i}-J_{i}\right) / 2,\left(D_{i}-J_{i}\right)\right)$. This is evident from the fact that in the worst-case task releases occur at $t=0, \quad t=m T_{j}-J_{j}$ for $m=1,2,3 \ldots$

There are two cases to consider to complete the proof:

1. $\tau_{i}$ is schedulable with $R_{i} \geq\left(D_{i}-J_{i}\right) / 2$. In this case, the initial value $\left(D_{i}-J_{i}\right) / 2$ is less than the first solution of the recurrence relation, and so the equation is guaranteed to converge on $R_{i}$.

2. $\tau_{i}$ is schedulable with $R_{i}<\left(D_{i}-J_{i}\right) / 2$. In this case, as $\tau_{i}$ has completed execution by $\left(D_{i}-J_{i}\right) / 2$, only tasks in $h p(i)$ are able to execute after this point. As the longest priority level(i-1) busy period is known to be of length $R_{i-1} \leq R_{i} \leq\left(D_{i}-J_{i}\right) / 2$, a further idle instant must occur by $D_{i}-J_{i}$. The recurrence relation is therefore guaranteed to converge on some value $R_{i}^{U B}$, where $R_{i} \leq R_{i}^{U B} \leq D_{i}-J_{i}$

We now improve on the initial value given by Theorem 3 .

Theorem 4. The initial value $\left(D_{i}-J_{i}+C_{i}+B_{i}\right) / 2$ guarantees that the recurrence relation will converge to a solution $R_{i}^{U B}$, where $R_{i} \leq R_{i}^{U B} \leq D_{i}-J_{i}$ if $\tau_{i}$ is in fact schedulable. 
Proof. To prove the theorem, there are again two cases to consider:

1. $\tau_{i}$

is schedulable with $R_{i} \geq\left(D_{i}-J_{i}+C_{i}+B_{i}\right) / 2$. In this case, the initial value $\left(D_{i}-J_{i}+C_{i}+B_{i}\right) / 2$ is less than the first solution to the recurrence relation, and so the equation is guaranteed to converge on $R_{i}$

2. $\tau_{i}$ is schedulable with $R_{i}<\left(D_{i}-J_{i}+C_{i}+B_{i}\right) / 2$. In this case, the interference due to higher priority tasks in $R_{i}$ is at most $\left(D_{i}-J_{i}-C_{i}-B_{i}\right) / 2$, the additional time being accounted for by blocking $B_{i}$ and the execution time $C_{i}$ of task $\tau_{i}$ itself. Hence the longest possible priority level-(i-1) busy period comprising only execution of tasks in $h p(i)$ is of length $\left(D_{i}-J_{i}-C_{i}-B_{i}\right) / 2$. This means that there must exist a priority level-i idle instant in the interval between $\left(D_{i}-J_{i}+C_{i}+B_{i}\right) / 2$ and $\left(D_{i}-J_{i}+C_{i}+B_{i}\right) / 2+\left(D_{i}-J_{i}-C_{i}-B_{i}\right) / 2$ $=D_{i}-J_{i}$. The recurrence relation is therefore guaranteed to converge on some value $R_{i}^{U B}$, where $R_{i} \leq R_{i}^{U B} \leq D_{i}-J_{i}$

We note that as the initial values given by Theorems 1 to 4 may be larger than the exact worst-case response time $R_{i}$, these initial values cannot be used to calculate exact worst-case response times, only to provide an exact Boolean schedulability test.

Using the initial values given by Theorems 1 to 4 , the next value generated by the recurrence relation may, in some cases, be smaller than the initial value. If so, iteration can be terminated immediately, as the task is then known to be schedulable, with the value computed on the $1^{\text {st }}$ iteration providing an upper bound $R_{i}^{U B}$ on the worstcase response time.

\subsection{Example}

In this section, we use the simple example taskset described in Table 1 to illustrate the operation of the initial values given by Theorems 2 and 4 .

TABLE 2: INITIAL VALUES AND UPPER BOUND

\begin{tabular}{|c|c|c|c|c|c|c|c|}
\hline \multirow[b]{2}{*}{ Pri } & \multirow[b]{2}{*}{ C } & \multirow[b]{2}{*}{ D } & \multicolumn{3}{|c|}{ Initial values } & \multirow[b]{2}{*}{$R_{i}^{U B}$} & \multirow[b]{2}{*}{$R_{i}$} \\
\hline & & & $\mathbf{T}$ & (i) & (ii) & & \\
\hline 1 & 5 & 10 & 10 & - & - & 5 & 5 \\
\hline 2 & 25 & 100 & 100 & 95 & 62.5 & 75 & 50 \\
\hline 3 & 25 & 200 & 200 & 125 & 112.5 & 150 & 100 \\
\hline 4 & 30 & 1000 & 1200 & 850 & 515 & 795 & 360 \\
\hline 5 & 30 & 1200 & 1200 & 405 & 615 & 670 & 570 \\
\hline
\end{tabular}

Here we use the maximum of the two initial values: (i) $\left(D_{i}-J_{i}\right)-R_{i-1}^{U B}$ and (ii) $\left(D_{i}-J_{i}+C_{i}+B_{i}\right) / 2$ as a starting point for the recurrence relation. These values are shown in Table 2, along with the computed upper bound $R_{i}^{U B}$ on the worst-case response time of each task. These upper bounds were calculated in priority order. Note that the exact worst-case response times are not calculated by this method; however, they are shown for comparison purposes in the final column of the table.

We note that the large initial value of 850 provided for $\tau_{4}$ meant that the recurrence relation terminated on the first iteration, giving an upper bound $R_{4}^{U B}$ of 795 . In the case of $\tau_{5}$, the initial value of 615 enabled task schedulability to be determined in 5 iterations (compared with 7 or 10 iterations using the initial values discussed in Section 3).

\section{Schedulability test efficiency}

In this section, we outline three other methods of improving the efficiency of exact schedulability tests based on Response Time Analysis, aside from using appropriate initial values:

1. Using a sufficient schedulability test to quickly determine, on a task-by-task basis, if an exact schedulability calculation is required. This approach is only applicable to Boolean schedulability tests where exact response times are not required.

2. Alternative implementations of the recurrence relation. This approach is applicable to both Boolean schedulability tests and response time tests.

3. Checking task schedulability in reverse priority order. This approach seeks to identify unschedulable tasks early, reducing the amount of computation required when a taskset is unschedulable. This approach is also applicable to both exact Boolean schedulability tests and exact response time tests.

\subsection{Sufficent schedulability test}

The use of a suitable sufficient schedulability test on a task-by-task basis can in theory improve the efficiency of an exact Boolean schedulability test. If a task is schedulable according to the sufficient test, then exact schedulability does not need to be calculated, short circuiting a significant amount of computation. Note this approach is not appropriate if exact response times are required.

A number of simple sufficient schedulable tests have appeared in the literature. These include the Utilisation Bound [3], the Hyperbolic Bound [26], the Utilisation RBound [27], and the response time upper bound [18]. The complexity of applying these tests to $n$ tasks is $\mathrm{O}(n)-$ if applied at each priority level, $\mathrm{O}(n), \mathrm{O}(n \log n)$, and $\mathrm{O}(n)$ respectively.

In [18], Bini and Baruah introduced the response time upper bound, and compared its performance to that of the Utilisation Bound, the Hyperbolic Bound, and the Utilisation RBound. They showed that the performance of the response time upper bound was superior to the other sufficient tests for $n>10$ and also when the task period dispersion, given by $\max _{i}\left(T_{i} / T_{i-1}\right)$ was greater than 2 .

As we are interested in improving the performance of an exact Boolean schedulability test in those cases where it can require a large number of iterations, (typically large $n$ and a wide range of task periods), then we use the re- 
sponse time upper bound, introduced by Bini and Baruah and reproduced in Equation (14) below, as our sufficient schedulability test of choice.

$$
R_{i}^{u b}=\frac{C_{i}+\sum_{\forall j \in h p(i)} C_{j}\left(1-U_{j}\right)}{1-\sum_{\forall j \in h p(i)} U_{j}}
$$

It is interesting to note that, although using Equation (14) alone as a sufficient schedulability test results in poor performance for tasksets with high utilisation, there are still a significant number of individual tasks that are schedulable according to Equation (14), even if the taskset as a whole is not. This is illustrated by Figure 1 which shows the percentage of tasksets, all of which are schedulable according to an exact test, that are deemed schedulable using a sufficient test based on Equation (14). Whilst performance of the sufficient test rapidly tails off above $80 \%$ utilisation, the number of individual tasks deemed schedulable remains high (over $85 \%$ at $97.5 \%$ utilization). Note this data is based on averages over 10,000 tasksets for each utilisation level, with each taskset comprising 24 tasks and having a range of task periods spanning 4 orders of magnitude. See Section 6 for further details of the tasksets used.

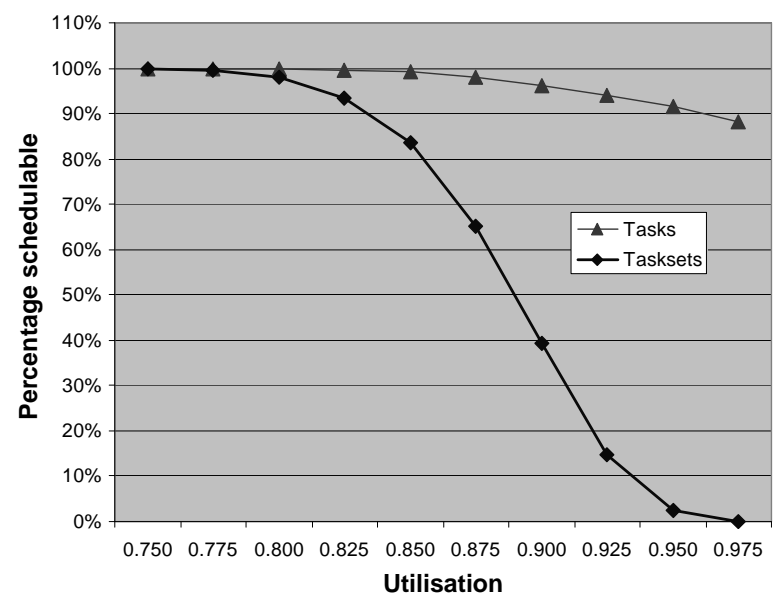

Figure 1: Percentage of tasks and tasksets that were deemed to be schedulable by the response time upper bound given in Equation (14), plotted against taskset utilisation.

\subsection{Algorithm implementations}

A standard implementation of the recurrence relation given by Equation (3) is shown in Figure 2 below. This $\mathrm{C}$ code fragment computes the response time of the task at priority $i$.

We note that in the standard implementation, although the computed response time may effectively increase each time line 9 is executed, these increases are not reflected in the value of the variable rprev passed to the ceiling() function until the next iteration of the while loop. With this in mind, the number of iterations of the for loop needed for convergence can be reduced by the alternative implemen- tation shown in Figure 3.

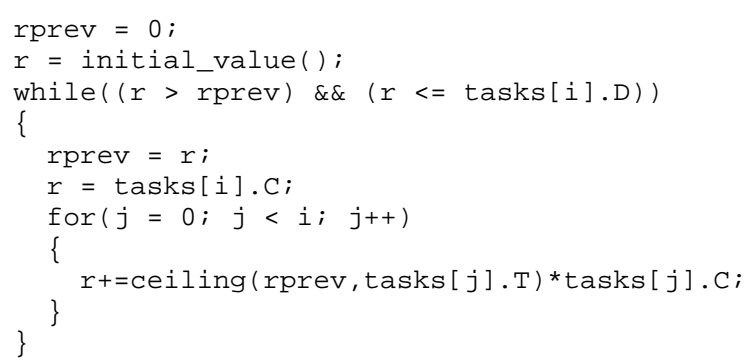

Figure 2: Standard implementation of the RTA algorithm.

The alternative implementation (Figure 3) records in the variable inter[j], the amount of interference due to task $j$, that has already been accounted for in the response time of task $i$. This facilitates incremental increases to the variable $r$ on each iteration of the second for loop (lines 1218).

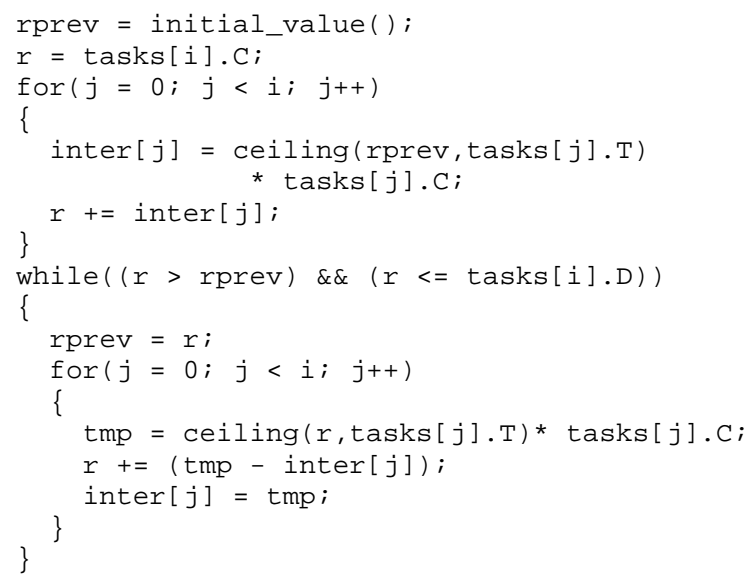

Figure 3: Incremental implementation of the RTA algorithm.

Note, in both implementations, it is assumed that the tasks are in priority order. Blocking factors and jitter terms are omitted from the code fragments for the sake of simplicity; however these can easily be included in either method.

\subsection{Order in which tasks are examined}

It can be argued that when a schedulability test is used as an online acceptance test, it does not matter how long the schedulability test takes to determine the schedulability of unschedulable tasksets. The schedulability test can always be suspended if it is taking too long, and the taskset deemed unschedulable (which is correct). What does matter is how long the test takes to determine the schedulability of schedulable tasksets. In this case suspending computation before an answer is available would mean wrongly classifying the taskset as unschedulable.

As schedulability of all tasks needs to be checked before a taskset can be shown to be schedulable, the amount of computation required by the schedulability test for schedulable tasksets is effectively independent of the order in which the tasks are examined. The initial value cal- 
culations may however require a particular ordering.

An alternative use of exact schedulability tests is as part of a design time tool, or online spare capacity allocation algorithm. In these cases, a binary search may be used to determine the maximum/minimum values of $C_{i}$ and $T_{i}$ that can be supported for each task or server. In this case, it is reasonable to expect a significant proportion, perhaps as many as $50 \%$, of the parameter sets put forward for testing to be unschedulable. Further, it is important to determine both schedulability and unschedulability efficiently, so that the higher level algorithm can make rapid progress towards its goal. In this case, checking schedulability in reverse priority order may be more effective. This is because lower priority tasks are more likely to be unschedulable than those of higher priority. Once a single task has been shown to be unschedulable then further computation can be abandoned, as the taskset as a whole is unschedulable.

Checking task schedulability in reverse priority order is only possible using some of the initial values discussed. The initial values given by Equations (5) and (7), and Theorems 1, 3 and 4, do not depend on the order in which task schedulability is checked, and so may be used when schedulability is determined in reverse priority order. By contrast, the initial values given by Equations (4), (6), and (11), and Theorem 2 rely on knowing the response time, or an upper bound on the response time, of the next highest priority task. These initial values cannot be used if task schedulability is checked in reverse priority order.

\section{Empirical investigation}

In this section, we describe an empirical investigation into the effectiveness of using the initial values introduced in Sections 3 and 4, the sufficient test, algorithm implementations and task orderings discussed in Section 5.

For ease of reference, Table 3 provides a summary of the initial values and algorithm options used in our experiments. In the remainder of this section and in Section 7 , we refer to the initial values and algorithm options used by these numbers, thus \#1 refers to the standard algorithm implementation given in Figure 2, using the default initial value $C_{i}$, whilst $\# 9$ refers to using a sufficient test based on Equation (14) to determine when exact analysis is required and then an exact test using the standard algorithm implementation, with the initial value given by the maximum of Equation (7), Theorem 2 and Theorem 4.

The experiments described in this section were performed on a PC, enabling results to be obtained for large numbers of randomly generated tasksets. In Section 7, we complement this data with execution time measurements, for a small number of tasksets, recorded on an embedded microprocessor.
TABLE 3: RTA OPTIONS: INITIAL VALUES AND ALGORITHMS

\begin{tabular}{|c|c|c|}
\hline & Initial Value & Algorithm \\
\hline$\# 1$ & Default & \multirow{8}{*}{$\begin{array}{l}\text { Standard algorithm (see } \\
\text { Figure 2). }\end{array}$} \\
\hline$\# 2$ & Equation (7) & \\
\hline$\# 3$ & Equation (6) & \\
\hline$\# 4$ & $\begin{array}{l}\text { Max of Equations (6) } \\
\text { and (7) }\end{array}$ & \\
\hline$\# 5$ & Equation (11) & \\
\hline$\# 6$ & Theorem 1 & \\
\hline$\# 7$ & Theorem 2 & \\
\hline$\# 8$ & Theorem 4 & \\
\hline$\# 9$ & $\begin{array}{l}\text { Max of Equation (7) } \\
\text { and Theorems } 2 \text { and } 4\end{array}$ & $\begin{array}{l}\text { Sufficient test then } \\
\text { standard algorithm. }\end{array}$ \\
\hline$\# 10$ & Default & \multirow{2}{*}{$\begin{array}{l}\text { Incremental algorithm } \\
\text { (see Figure 3). }\end{array}$} \\
\hline \#11 & Equation (11) & \\
\hline$\# 12$ & Default & \multirow{2}{*}{$\begin{array}{l}\text { Standard algorithm, } \\
\text { reverse priority order. }\end{array}$} \\
\hline$\# 13$ & Equation (7) & \\
\hline$\# 14$ & Default & \multirow{5}{*}{$\begin{array}{l}\text { Sufficient test, then } \\
\text { standard algorithm, } \\
\text { reverse priority order. }\end{array}$} \\
\hline$\# 15$ & Equation (7) & \\
\hline$\# 16$ & Theorem 1 & \\
\hline$\# 17$ & Theorem 4 & \\
\hline$\# 18$ & $\begin{array}{l}\text { Max of Equation (7) } \\
\text { and Theorems } 1 \text { and } 4\end{array}$ & \\
\hline
\end{tabular}

The task parameters used in our experiments were randomly generated as follows: Of the $n$ tasks in each taskset, $n / M$ tasks were assigned periods, according to a uniform random distribution, from each of the $M$ order of magnitude ranges used (e.g. 1000-10000, 10000-100000, 100000-1000000 etc). This was done both to replicate the type of period distributions found in commercial real-time systems, and also to enable an investigation into how the efficiency of response time analysis depends upon the range of task periods. In all cases, task deadlines were set equal to their periods.

For each utilisation level studied, from $75 \%$ to $97.5 \%$ in increments of $2.5 \%$, the UUniFast algorithm [15] was used to determine the distribution of overall utilisation between the tasks, and hence task computation times given the selected task periods. 10,000 tasksets were generated for each utilisation level, equating to some 100,000 tasksets in all.

We used the number of ceiling operations as a performance metric to compare the different approaches and as a simple proxy for schedulability test execution time.

The results of Experiments 1-3 below record the number of ceiling operations required to determine the schedulability of schedulable tasksets. This avoids skewing the data, due to the significant numbers of unschedulable tasksets that occur at high levels of utilisation. Experiment 4 specifically examines this effect and shows how the time taken to determine the schedulability of unschedulable tasksets depends upon the order in which tasks are checked.

\subsection{Experiment 1}

This experiment investigated the efficiency of exact schedulability tests used to determine the feasibility of 
tasksets comprising 24 tasks with periods spanning 4 orders of magnitude, and overall utilisations varying from $75 \%$ to $97.5 \%$.

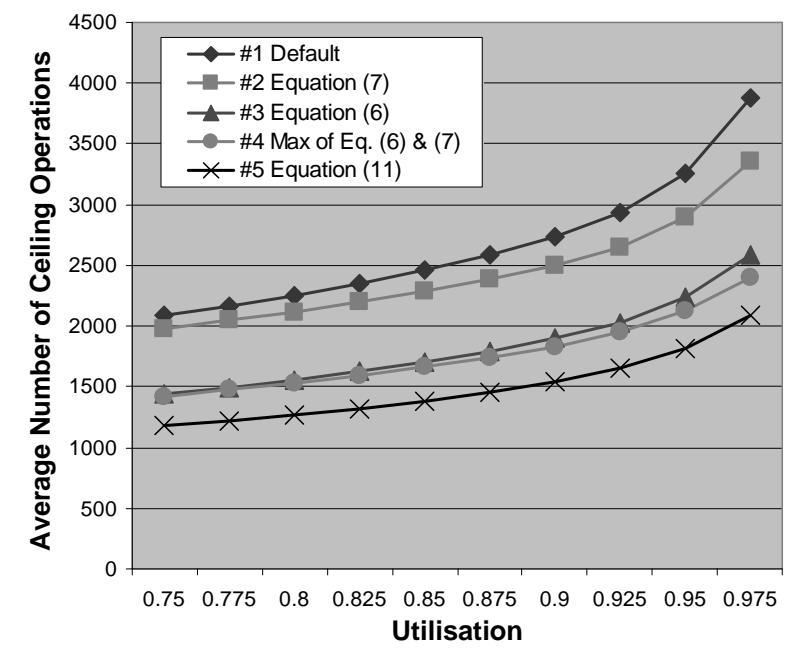

Figure 4: Average number of "ceiling operations" required by exact response time tests, plotted against taskset utilisation. Data is for the standard RTA algorithm implementation, using the initial values described in Section 3.

Figure 4 shows the average number of ceiling operations required using the standard algorithm implementation and the initial values described in Section 3. This graph shows that using the new initial value $\# 5$, given by Equation (11), results in the schedulability test requiring the least number of ceiling operations. However, this result is slightly misleading. Determining this initial value is more complex than the others, effectively requiring $n(n+1) / 2$ additional ceiling operations (300 in the case of 24 tasks). This reduces performance to a similar level to that obtained by using initial value $\# 4$, given by the maximum of Equations (6) and (7).

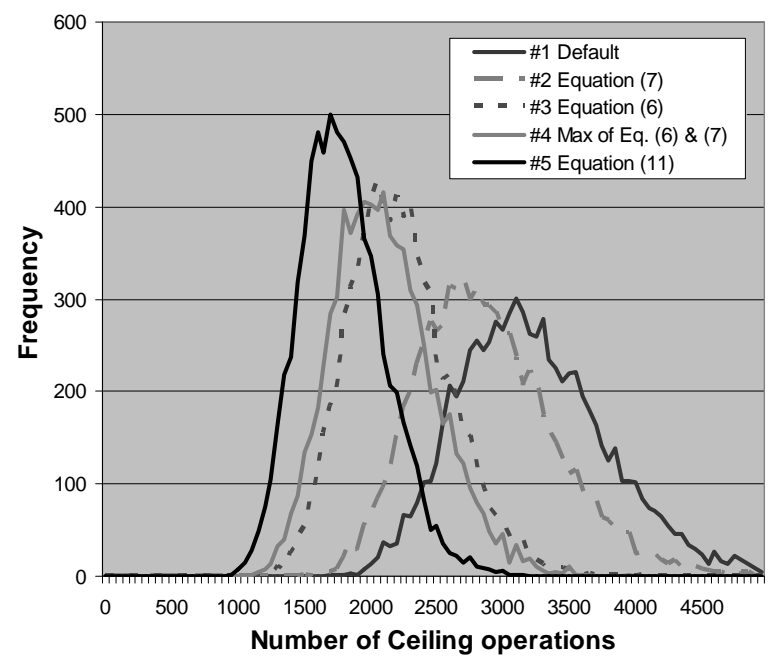

Figure 5: Frequency distribution of the number of "ceiling operations" required by exact response time tests, starting with the initial values described in Section 3. Data is for 10,000 tasksets with $95 \%$ utilisation.
Figure 5 illustrates the frequency distribution of the number of ceiling operations required by the schedulability test for each of the 10,000 tasksets with $95 \%$ utilisation. The graph shows that using initial value $\# 5$ given by Equation (11) results in, a smaller maximum, a narrower frequency distribution, and a smaller average than \#1, the default initial value.

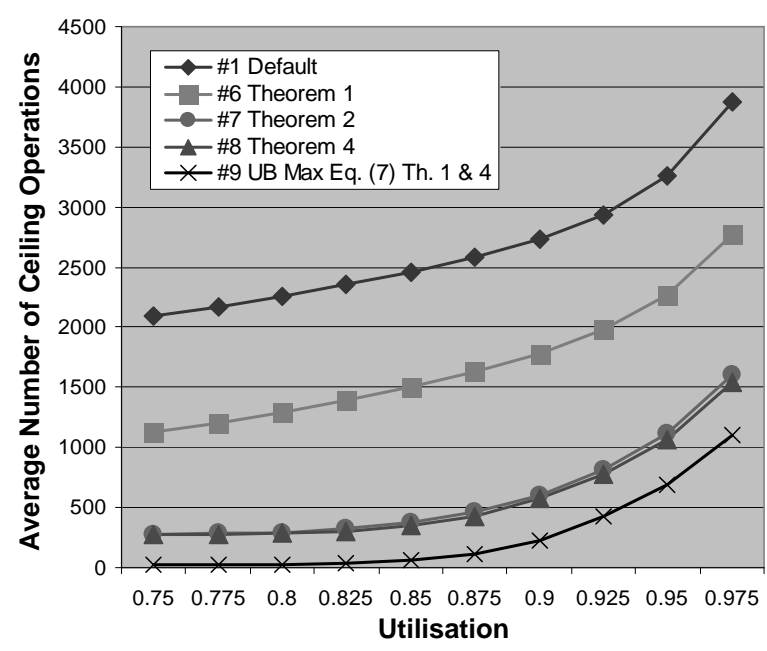

Figure 6: Average number of "ceiling operations" required by exact Boolean schedulability tests, plotted against taskset utilisation. Data is for the standard RTA algorithm implementation, using the initial values described in Section 4.

Figure 6 is similar to Figure 4; however, it shows the average number of ceiling operations required using the initial values described in Section 4. Figure 6 shows that using the two new initial values \#7 and \#8 given by Theorems 2 and 4 result in significantly improved performance. For utilisation levels from $75-90 \%$, the algorithm requires just 1 or 2 iterations of the while loop on average. This is because in many cases, the value computed on the $1^{\text {st }}$ iteration is less than the initial value, resulting in an immediate exit.

Finally, using the response time upper bound given by Equation (14), to determine if an exact schedulability computation is needed, combined with the maximum of the initial values given by Equation (7) and Theorems 1 and 4, results in excellent performance (\#9 on the graph). Using this approach, it is very rare that the exact schedulability computation is required at utilisation levels below about $85 \%$. (Recall that Figure 1 shows that on average, at $97.5 \%$ utilisation, only $12 \%$ of the tasks require an exact schedulability computation).

Figure 7 illustrates the frequency distribution of the number of ceiling operations required by the schedulability test for each of the 10,000 tasksets with $95 \%$ utilisation. This graph shows that $\# 9$, using the response time upper bound given by Equation (14) to determine if an exact schedulability computation is needed, combined with the maximum of the initial values given by Equation (7) and Theorems 1 and 4, results in a significantly lower 
maximum number of ceiling operations, narrower distribution, and smaller average than the default initial value \#1. Note 194 tasksets were schedulable using the sufficient test alone. This accounts for the initial peak in line \#9.

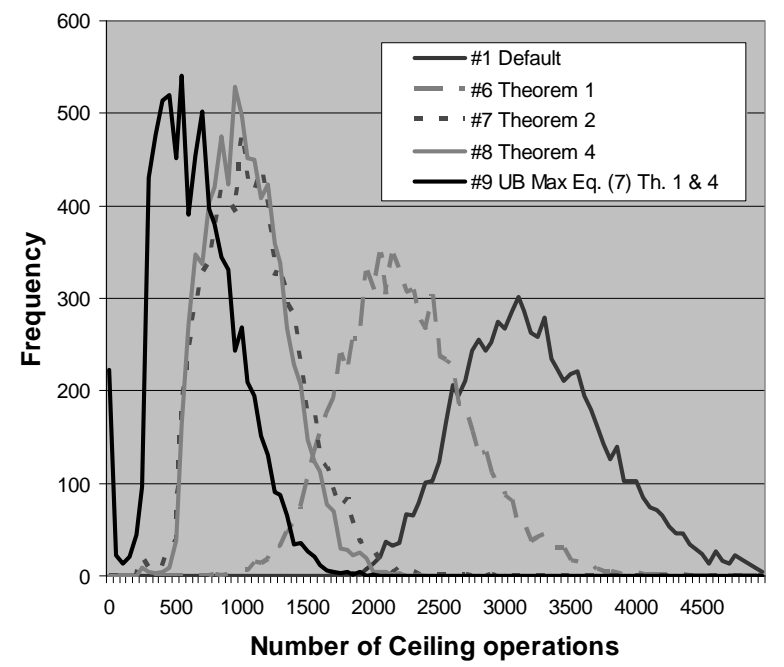

Figure 7: Frequency distribution of the number of "ceiling operations" required by exact Boolean schedulability tests, starting with the initial values described in Section 4. Data is for 10,000 tasksets with $95 \%$ utilisation.

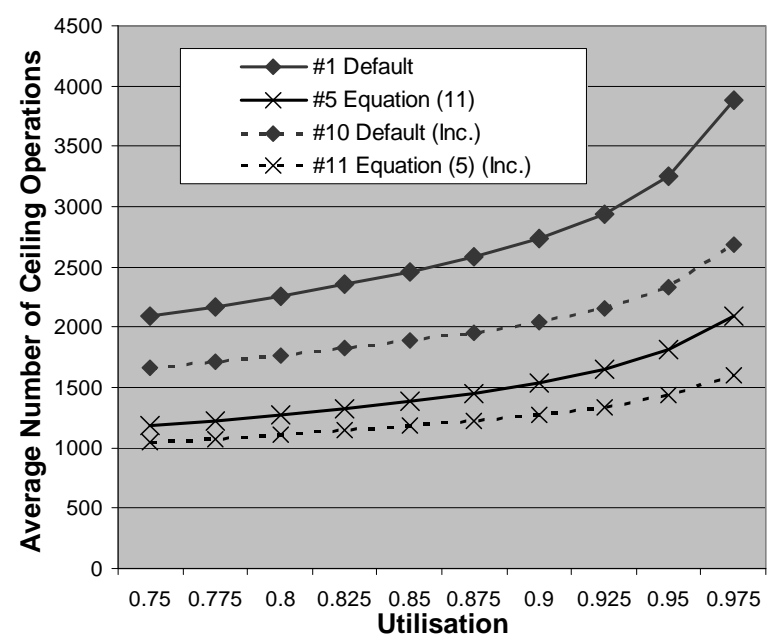

Figure 8: Comparison between the standard and incremental implementations of the RTA algorithm.

Figure 8 compares the standard and incremental algorithm implementations described in Section 5.2 for the default initial value $C_{i}$ and for the initial value given by Equation (11). This graph shows that the alternative, incremental implementation converges significantly faster (see lines \#10 and \#11 on the graph compared with \#1 and \#5 respectively). We note however, that there is more computation on each inner loop iteration of the incremental implementation. We return to this point in Section 7.

\subsection{Experiment 2}

This experiment was similar to Experiment 1, only instead of varying the utilisation of the tasksets overall utilisation was fixed at $95 \%$, and the range of task periods was varied from 2 to 6 orders of magnitude.

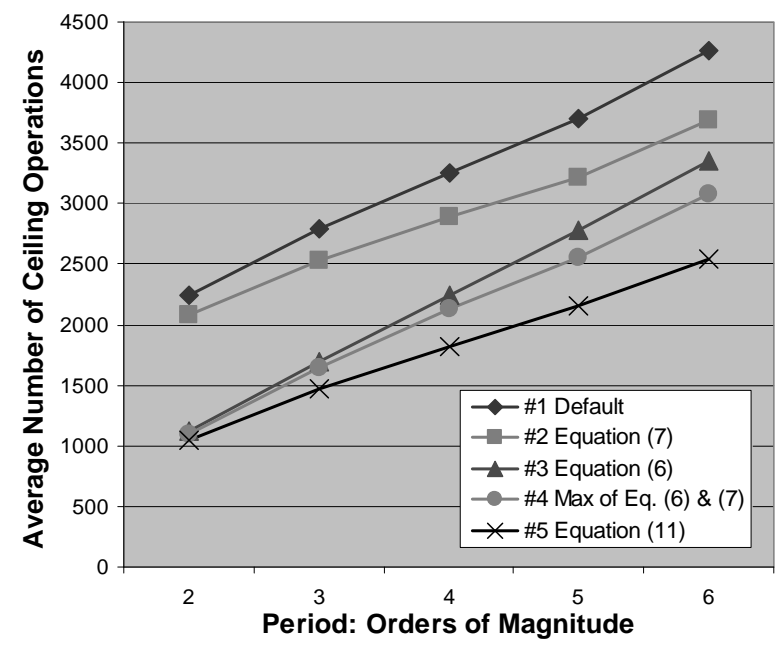

Figure 9: Average number of "ceiling operations" required by exact response time tests, plotted against the number of orders of magnitude range of task periods, for tasksets with 95\% utilisation. Data is for the standard RTA algorithm implementation, using the initial values described in Section 3.

Figure 9 illustrates that for all of the initial values discussed in Section 3 (that can be used to compute exact response times), the average number of ceiling operations required increases approximately linearly with the number of orders of magnitude spanning task periods. We note that the increase is slower for the new initial value \#5 given by Equation (11). Adjusting for the extra cost of determining this initial value $(+300)$, it provides higher performance for larger ranges of task periods ( $>4$ orders of magnitude) and lower performance for smaller ranges $(<4$ orders of magnitude).

Figure 10 illustrates that for the initial values discussed in Section 4 (that can be used to compute exact schedulability but not exact response times, i.e. \#6, \#7 and \#8); the average number of ceiling operations required remains approximately constant, irrespective of the range of tasks periods. This is an interesting result as it shows that these initial values are particularly useful in reducing the execution time of the schedulability test in just those cases where the task parameters (range of task periods and computation time values) tend to increase the number of iterations required.

Using the response time upper bound given by Equation (14) to determine whether an exact schedulability computation is required is highly effective, when combined with the maximum of the initial values given by Equation (7) and Theorems 1 and 4, (\#9 on the graph). In particular, the upper bound becomes more accurate as the range of task periods increases, resulting in a decrease in 
the number of times that an exact schedulability computation is required, and consequently a decrease in the average number of ceiling operations required.

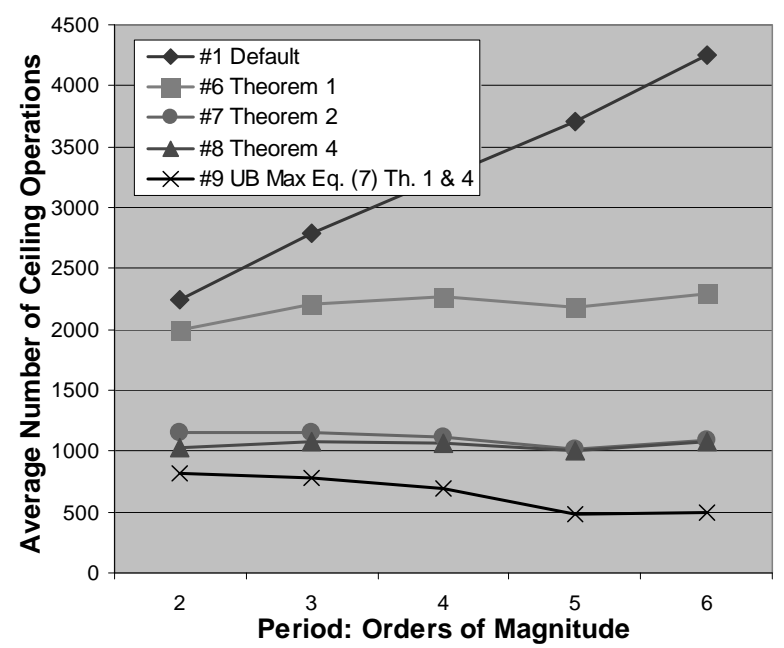

Figure 10: Average number of "ceiling operations" required by exact Boolean schedulability tests, plotted against the number of orders of magnitude range of task periods, for tasksets with $95 \%$ utilisation. Data is for the standard RTA algorithm implementation, using the initial values described in Section 4.

\subsection{Experiment 3}

In this experiment, we varied the number of tasks from 8 to 256 , with the range of task periods fixed at 4 orders of magnitude, and the taskset utilisation fixed at $95 \%$.

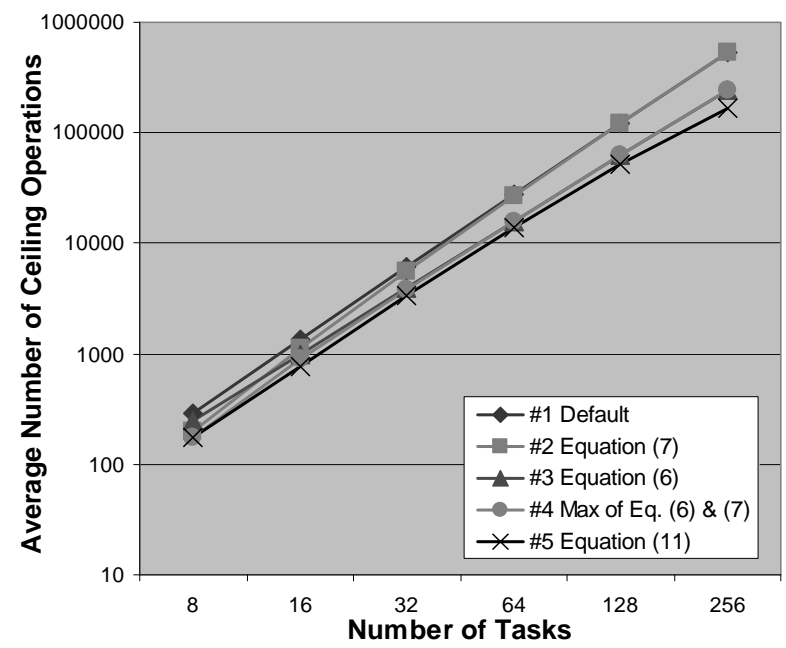

Figure 11: Average number of "ceiling operations" required by exact response time tests, plotted against the number of tasks in each taskset, for tasksets with $95 \%$ utilisation. Data is for the standard RTA algorithm implementation, using the initial values described in Section 3.

Figure 11 and Figure 12 show that the average number of ceiling operations increases roughly as the square of the number of tasks. Note the log. scale on these graphs.

Figure 13 shows how the average number of ceiling operations required for each of the initial values given in Section 3 relates to the average number of ceiling operations required when starting with the default initial value. We note that as the number of tasks increases, the utilisation and thus execution time of the individual tasks becomes smaller. This tends to make initial value \#3 based on Equation (6) more accurate and initial value \#2 based on Equation (7) less accurate. The initial value \#5, given by Equation (11), performs progressively better for a larger number of tasks. This is because for large $n$, typically at least one of the $n$ potential initial values generated by Equation (11) is a close approximation to the exact response time.

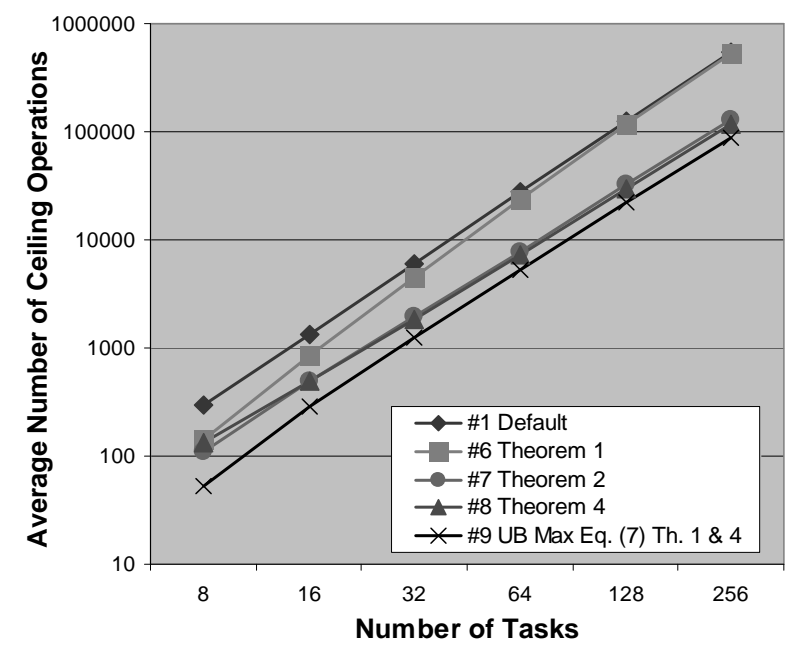

Figure 12: Average number of "ceiling operations" required by exact Boolean schedulability tests, plotted against the number of tasks in each taskset, for tasksets with 95\% utilisation. Data is for the standard RTA algorithm implementation, using the initial values described in Section 4.

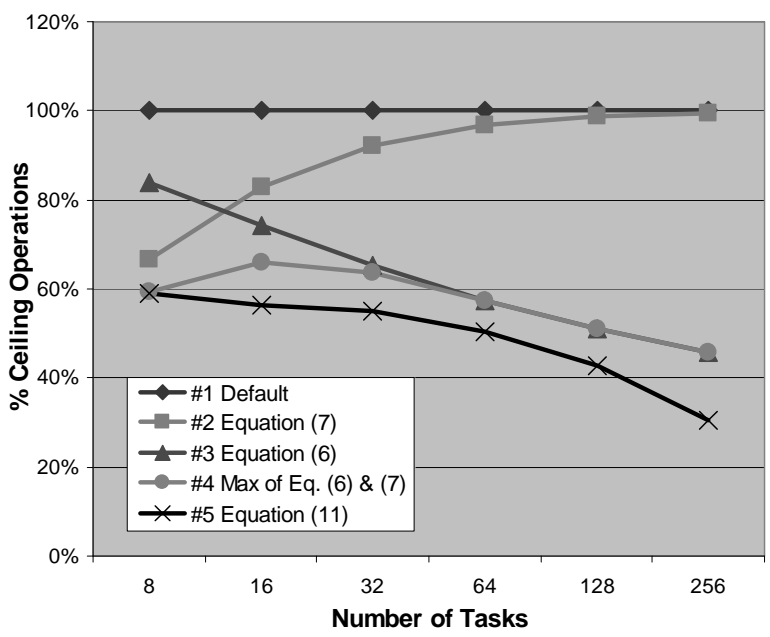

Figure 13: Performance of exact response time tests relative to the default approach, plotted against the number of tasks in each taskset, for tasksets with $95 \%$ utilisation. Data is for the standard RTA algorithm implementation, using the initial values described in Section 3.

Figure 14 shows how the average number of ceiling operations required for each of the initial values given in Section 4 relates to the average number of ceiling opera- 
tions required when starting with the default initial value. We note that as the number of tasks increases, the differences between the deadlines of two tasks with adjacent priorities becomes progressively smaller. Thus initial value \#6 based on deadline difference performs poorly with an increasing number of tasks. By contrast, the fact that individual task execution times tend to decrease with an increasing number of tasks (for the same overall utilisation and period distribution) means that the other initial values work progressively better for larger numbers of tasks.

Using the response time upper bound, Equation (14), to determine whether an exact schedulability computation is required is once again highly effective. Combined with the maximum of the initial values given by Equation (7) and Theorems 1 and 4, (\#9 on the graph), it reduces the number of ceiling operations required to around $20 \%$ of the default approach.

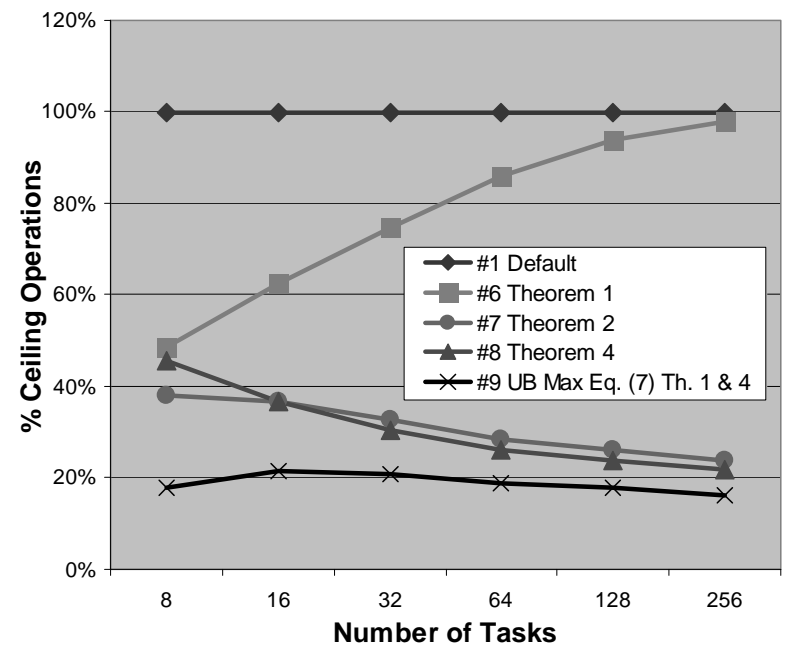

Figure 14: Performance of exact Boolean schedulability tests relative to the default approach, plotted against number of tasks in each taskset, for tasksets with $95 \%$ utilisation. Data is for the standard RTA algorithm implementation, using the initial values described in Section 4.

\subsection{Experiment 4}

In this experiment, we examined the hypothesis that checking tasks in reverse priority order helps identify unschedulable tasks early and thus decreases the average number of ceiling operations required by the schedulability test to identify unschedulable tasksets.

This experiment used the same tasksets as Experiment 1. Recall that these tasksets comprised 24 tasks with periods spanning 4 orders of magnitude, for total taskset utilisations varying from $75 \%$ to $97.5 \%$.

Figure 15 shows the average number of ceiling operations required to determine schedulability and exact response times using the default initial value, for (a) schedulable tasksets only, (b) all tasksets, schedulable and unschedulable, (c) all tasksets - checking schedulability in reverse priority order, (d) unschedulable tasksets only, and (e) unschedulable tasksets only - checking schedulability in reverse priority order. It is clear from the graph that for the tasksets studied; checking schedulability in reverse priority order identified unschedulable tasksets more efficiently, reducing the average number of ceiling operations required.

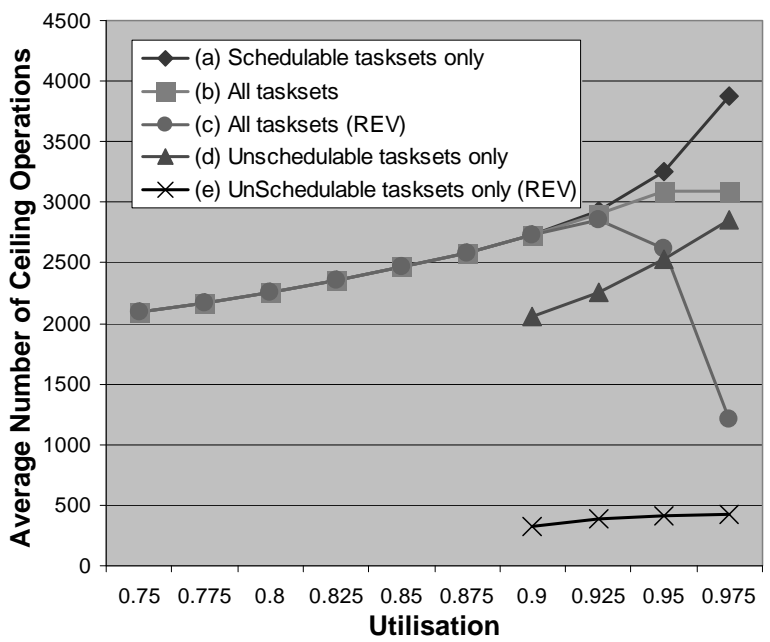

Figure 15: Average number of "ceiling operations" required by exact response time tests, plotted against taskset utilisation. Data is for the standard RTA algorithm implementation (forward and reverse priority order) using the default initial value.

Note that out of the 10,000 tasksets at each utilisation level, the percentage that were unschedulable was zero up to $85 \%$ utilisation, then $0.01 \%, 0.2 \%, 3.3 \%, 26.5 \%$ and $77.4 \%$ at utilisation levels of $87.5 \%, 90 \%, 92.5 \%, 95 \%$, and $97.5 \%$ respectively. (This is why Figure 15 shows data for unschedulable tasksets only from $90 \%$ utilisation upwards). Partly, this is because the tasks all had deadlines equal to their periods $(D=T)$. For systems with $D<$ $T$, unschedulability is to be expected at lower utilisation levels.

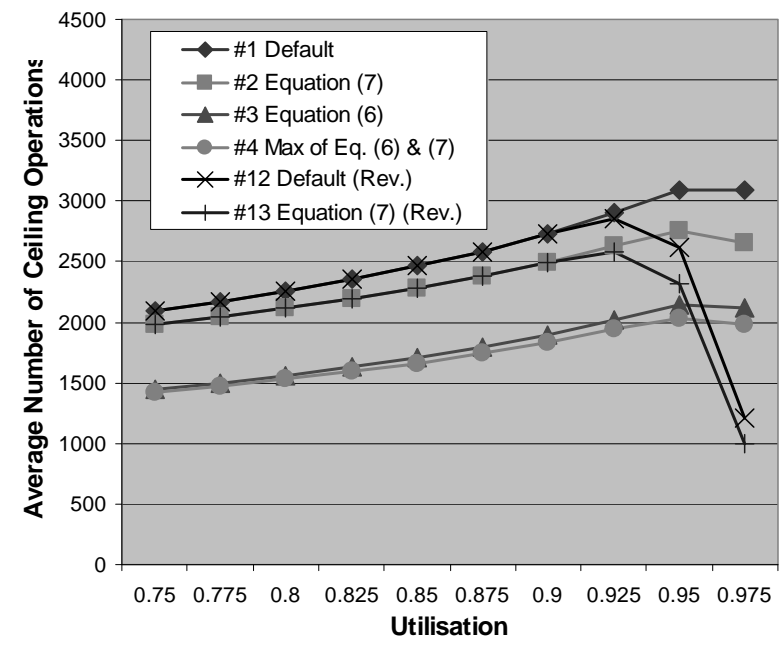

Figure 16: Average number of "ceiling operations" required by exact response time tests, plotted against taskset utilisation. Data is for the standard RTA algorithm implementation (forward and 
reverse priority order) using the initial values described in Section 3.

Figure 16 shows the average number of ceiling operations required to determine schedulability and exact response times, using the initial values discussed in Section 3 . In this respect, Figure 16 is similar to Figure 4; however, whereas the data for Figure 4 is from schedulable tasksets only. Figure 16 uses data from all tasksets, both schedulable and unschedulable. In addition, options \#12 and \#13 in Figure 16 show the effect of checking task schedulability in reverse priority order. Note this is possible only for those initial values that are not dependent on knowing the response time of the next highest priority task.

It is clear from Figure 16 that at very high utilisation levels $(>95 \%)$, where there is a high probability that a taskset will be unschedulable, it is worthwhile checking task schedulability in reverse priority order. This enables the schedulability test to terminate early when a low priority task is found to be unschedulable. Note that at lower utilisation levels, it is appropriate to use other more effective initial values, for example \#4, given by the maximum of Equations (6) and (7).

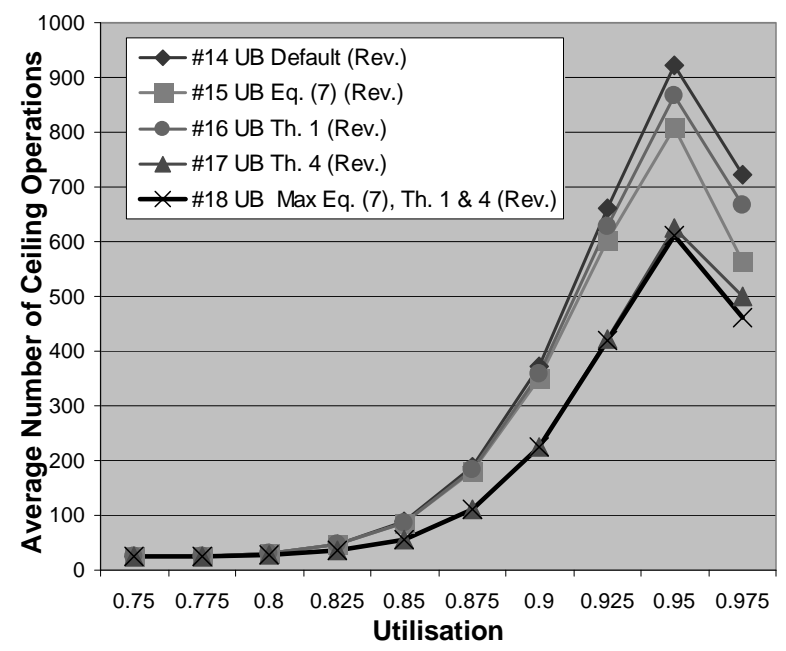

Figure 17: Average number of "ceiling operations" required by exact Boolean schedulability tests, plotted against taskset utilisation. Data is for the standard RTA algorithm implementation (reverse priority order), using a sufficient test to determine when exact schedulability computation is required, and the initial values described in Section 4.

Figure 17 shows the average number of ceiling operations required to determine taskset schedulability, via a Boolean schedulability test, using the default initial value (\#14), the initial value given by Equation (7) (\#15), and the initial values given by Theorems 1 and 4 (\#16 and $\# 17$ ), and finally the maximum of the initial values given by Theorems 1 and 4 and Equation (7) (\#18). In each case, the response time upper bound was used on a task-by-task basis to determine if an exact schedulability computation was required. Also in each case, task schedulability was checked in reverse priority order.
Figure 17 shows that examining tasks in reverse priority order results in a reduction in the average number of ceiling operations required at very high utilisation levels $(>95 \%)$. This is because it is more likely that the low priority tasks will be unschedulable and hence the schedulability test can terminate earlier. Figure 17 also provides further evidence that the response time upper bound is highly effective in reducing the number of ceiling operations required at lower utilisation levels $(<85 \%)$.

\section{Execution time analysis}

In the previous section, we evaluated the efficiency of the exact RTA schedulability test, given various initial values, by counting the number of ceiling operations required for convergence. In this section, we look in more detail at the execution time of the algorithms, by running them on an embedded microprocessor.

Our test environment comprised a 32-bit PowerPC (MPC555) development board, clocked at 20MHz, with 4Mbytes of external SDRAM. Execution time information was obtained via the RapiTime worst-case execution time analysis toolset [24]. RapiTime was used to automatically instrument the code, capture timing traces, and produce a report of function execution times. The schedulability test algorithms were coded in $\mathrm{C}$, and compiled using the GNU $\mathrm{C}$ compiler, using optimisation level 2 (option -O2).

Using the embedded environment, it was only possible to carry out a limited number of experiments. We therefore confined our investigation to 3 specific tasksets, with utilisations of $75 \%, 85 \%$ and $95 \%$ respectively. The tasksets selected comprised 24 tasks, with periods distributed across a range spanning 4 orders of magnitude. These tasksets were worst-case, in the sense that of all 10,000 tasksets generated for each utilisation level, the ones selected required the largest number of ceiling operations to determine schedulability, starting with the default initial value of $C_{i}$. In this case, the numbers of ceiling operations required were 2956, 3959, and 6324 respectively.

TABLE 4: EXECUTION TIME MEASUREMENTS

\begin{tabular}{llllll}
\hline & \# & \multicolumn{2}{c}{ Clock Cycles } \\
Initial Value & Ops. & $\begin{array}{l}\text { Sched. } \\
\text { value }\end{array}$ & test & Total & \% \\
\hline \#1 Default & 6324 & 1371 & 163186 & 164557 & 100.0 \\
\#2 Equation (7) & 5724 & 4390 & 147751 & 152141 & 92.5 \\
\#3 Equation (6) & 3867 & 1688 & 100086 & 101774 & 61.9 \\
\#4 Max Equa- & & & & & \\
tions (6) and (7) & 3611 & 5179 & 93541 & 98720 & 60.0 \\
\#5 Equation (11) & 3094 & 24548 & 80275 & 104823 & 63.7 \\
\#6 Theorem 1 & 4357 & 1688 & 112494 & 114182 & 69.4 \\
\#7 Theorem 2 & 1609 & 1688 & 41962 & 43650 & 26.5 \\
\#8 Theorem 4 & 1573 & 1587 & 41010 & 42597 & 25.9 \\
\# 9 Max of Eq. & & & & & \\
(7), Theorems 2 & & 3051 & & & \\
and 4 & 722 & +615 & 18516 & 22182 & 13.5 \\
\hline
\end{tabular}


For the taskset with 95\% utilisation, Table 4 records: the number of ceiling operations required, the execution time in clock cycles to determine each initial value used, the execution time of the schedulability test (not including calculation of the initial value), the overall execution time of the schedulability test, and finally the percentage execution time with respect to the default approach.

Note that in the last row of the table, the data should be interpreted as follows: the response time upper bound (sufficient test) was computed for all 24 tasks, this took 3051 clock cycles in total (including looping over the tasks), as the 22 highest priority tasks were shown to be schedulable by this sufficient test, initial values were only computed for the two lowest priority tasks, this took 615 clock cycles. Finally, exact analysis of these two tasks took 722 ceiling operations, corresponding to 18516 clock cycles. The overall execution time of the schedulability test was 22182 clock cycles, some $13.5 \%$ of the time for the default approach.

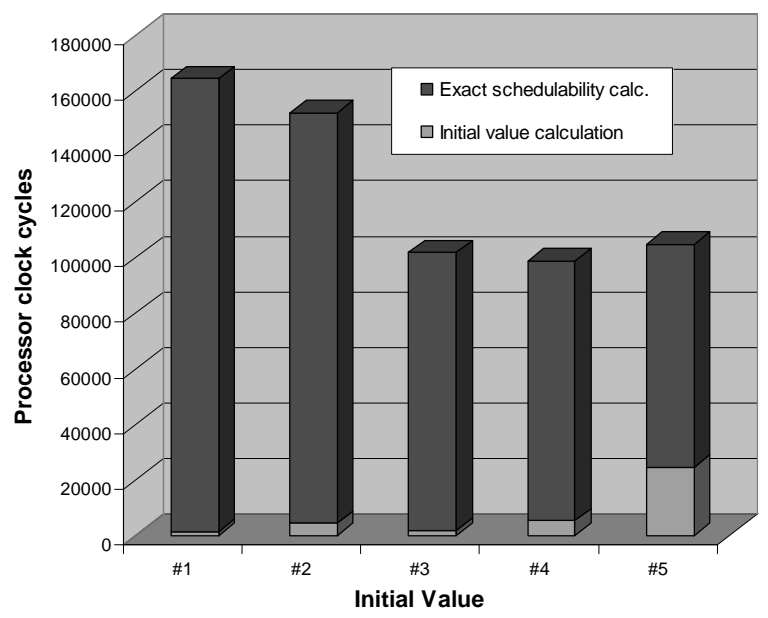

Figure 18: Execution times for exact response time tests, starting with the initial values described in Section 3. Data is for a single taskset with 95\% utilization, standard RTA algorithm implementation.

Figure 18 plots the data from Table 4, showing the execution time in clock cycles of the standard RTA schedulability test implementation, using the lower bound initial values described in Section 3. From the graph, it is clear that although the schedulability test itself is faster using initial value \#5 given by Equation (11), when the overheads of computing this initial value are included, its performance is similar to or slightly worse than that achieved by using initial value \#4 given by the maximum of Equations (6) and (7).

Figure 19 also plots the data from Table 4, showing the execution time in clock cycles of the standard RTA schedulability test implementation using the initial values described in Section 4. In this case, it is clear that the new deadline dependent initial values $\# 7$ and $\# 8$ given by Theorems 2 and 4 represent a significant reduction in exe- cution time compared with previous approaches. Taking the maximum of these two deadline dependent initial values and that given by Equation (7), and using the response time upper bound given by Equation (14) to determine when an exact schedulability calculation is required (\#9 on the graph), reduces the overall execution time of the schedulability test by a factor of 7.5 with respect to the default initial value (\#1).

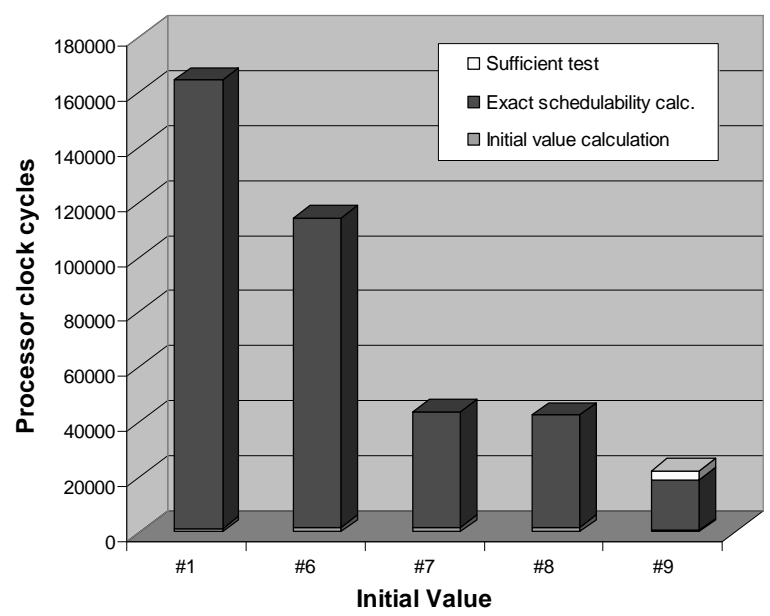

Figure 19: Execution times for exact Boolean schedulability tests, starting with the initial values described in Section 4. Data is for a single taskset with 95\% utilization, standard RTA algorithm implementation.

We also recorded a set of execution time measurements for the incremental schedulability test implementation described in Figure 3 in Section 5.2. For the 95\% utilisation taskset, we found that the number of inner loop iterations was reduced to between $68 \%$ and $81 \%$ of the number required by the standard implementation, dependent on the initial value used. Despite this, the overall execution time was between $104 \%$ and $121 \%$ of the times recorded for the standard implementation. On this particular processor, the extra overheads in the inner loop outweighed the reduction in the number of loop iterations. The execution time for one inner loop iteration was 26 clock cycles for the standard implementation and 39 clock cycles for the incremental approach.

We note that this finding is representative only of the specific microprocessor/compiler combination used; we expect that for some combinations the incremental approach would be more efficient, whilst for others the standard approach is better.

\section{Recommendations}

In this section, we provide recommendations to engineers tasked with the problem of implementing exact schedulability tests in the following areas:

1. as an online acceptance test,

2. as part of an online spare capacity allocation algorithm or, 
3. as part of an offline design-time tool.

Recommendation 1: We recommend using the Response Time Analysis based schedulability tests described in this paper, rather than the alternative Hyperplanes Exact Test (HET) described in [19].

Rationale: We found that the performance of the HET algorithm is extremely sensitive to the range of task periods, and that in practice it is significantly outperformed by the RTA algorithm. See the Appendix for further details.

The most important consideration when choosing how best to implement an exact RTA based schedulability test is whether exact response times are required, or if a simple Boolean (schedulable/unschedulable) result will suffice.

Recommendation 2: If exact response times are not required, then we recommend the use of an appropriate sufficient test, such as the response time upper bound given by Equation (14), to determine whether to perform exact schedulability analysis on a task-by-task basis.

Rationale: Our experiments showed that even for tasksets with a very high utilisation, a significant number of individual tasks were schedulable according to the response time upper bound, and thus exact schedulability analysis for these tasks was unnecessary. Using this sufficient test to determine when exact analysis was required resulted in a significant improvement in efficiency.

Recommendation 3: If exact response times are not required, then we recommend using an initial value corresponding to the maximum of the values given by Equation (7) and Theorems 2 and 4, as a starting point for the recurrence relation (Equation (2)).

Rationale: Whilst this initial value does not guarantee that the recurrence relation will determine the exact worstcase response time, it does result in an exact schedulability test. Further, the number of operations required for convergence was found to be significantly lower using this initial value than using others that lead to exact response times.

Recommendation 4: If exact response times are required, then we suggest using the initial value given by Equation (11), or alternatively the initial value given by the maximum of Equations (4) and (5).

Rationale: Whilst the overheads of computing the initial value given by Equation (11) mean that it can typically be expected to provide broadly similar overall performance to that achieved starting with the maximum of Equations (4) and (5), in the circumstances where the recurrence relation tends to require a large number of ceiling operations for convergence (.i.e. large numbers of tasks, and/or a wide spread of task periods), then this initial value results in superior performance.

Recommendation 5: If the schedulability test is used as an online admission test, then we recommend checking task schedulability in priority order.

Rationale: When an exact schedulability test is used as an online admission test, then what is important is how long it takes to determine that a schedulable taskset is in fact schedulable. The time taken to determine that an unschedulable taskset is in fact unschedulable is of little consequence, for the reasons discussed in section 5.3. Checking task schedulability in priority order enables the most effective initial values to be used.

Recommendation 6: If the schedulability test is used as part of an online spare capacity allocation algorithm, or as part of a design-time tool, then we recommend considering checking task schedulability in reverse priority order.

Rationale: When an exact schedulability test is used as part of an online spare capacity allocation algorithm, or as part of a design-time tool, then it may be that a significant proportion of the task parameter sets considered represent unschedulable tasksets. In this case, efficiency can be improved by recognizing those unschedulable tasksets as soon as possible. This is best done by starting at the lowest priority. We note however, that checking task schedulability in reverse priority order effectively precludes the use of certain initial values, in particular, those given by Theorem 2, and Equations (4), (6) and (11).

Recommendation 7: Although the alternative, incremental implementation of the recurrence relation given in Figure 3 could possibly be more efficient for some compiler/microprocessor combinations, we recommend using the standard implementation given in Figure 2.

Rationale: The standard implementation is easier to code, takes up less code space, and is likely to be faster on more advanced processors due to the fact that it does not need to write to memory on each iteration of the inner loop.

\section{Summary and conclusions}

In this paper, we examined how the efficiency of schedulability tests based on the Response Time Analysis recurrence relation can be improved via:

1. The use of appropriate initial values.

2. Using a sufficient test to determine when calculation of exact schedulability is required.

3. Using an incremental algorithm implementation.

4. Examining task schedulability in reverse priority order.

We demonstrated the effectiveness of these approaches via empirical investigations on both a PC and on a realtime embedded micro-processor; a PowerPC MPC555. We then used these results to make a series of recommendations to Engineers tasked with implementing exact schedulability tests for; on-line acceptance tests, on-line spare capacity allocation, and off-line, design-time tools. 


\subsection{Contribution}

The main contributions of this paper are as follows:

1. Introducing a new family of initial values that can, in some cases, be used to improve schedulability test performance, when it is necessary to calculate the exact response time of each task.

2. Deriving improvements to the "deadline dependent" initial values introduced in [17], that are effective in increasing algorithm performance when an exact schedulability test is required, but upper bounds on response times will suffice (i.e. when exact response times are not required).

3. Extending the initial values introduced in [17] to account for blocking factors and release jitter. Removing these limitations makes it possible, for the first time, to use these initial values in the analysis of real-world systems.

4. Illustrating the efficiency improvements possible via using the response time upper bound developed in [18], to determine, on a task-by-task basis, if an exact schedulability computation is required.

5. Showing, in the appendix, that the performance of the Hyperplanes Exact Test [19] is extremely sensitive to the range of task periods, severely limiting the usefulness of this method when applied to real-world systems.

\subsection{Conclusion and future work}

The research presented in this paper showed that our approach of using the response time upper bound to determine when to compute exact schedulability, and new initial values as an advanced starting point, significantly reduces the execution time of exact schedulability tests based on Response Time Analysis. We intend to implement an on-line schedulability test, and spare capacity allocation algorithm based on this research, as part of the Frescor scheduling framework [23].

\section{Appendix: Comparison with Hyperplanes Exact Test}

In this appendix, we make some basic comparisons between the Response Time Analysis (RTA) methods described in this paper and the Hyperplanes Exact Test (HET) described in [19].

\section{Hyperplanes Exact Test}

In [6], Lehoczky et al. showed that for tasks that comply with the Liu and Layland [3] system model, exact schedulability of a task $\tau_{i}$ can be determined by inspecting the workload at all points $S_{i}$, corresponding to the releases of higher priority tasks between 0 and $T_{i}$.

$S_{i}=\left\{k T_{j}: j=1 . . i, k=1 . .\left\lfloor\frac{T_{i}}{T_{j}}\right\rfloor\right\}$

Thus $\tau_{i}$ is schedulable if and only if:
$L_{i}=\min _{t \in S_{i}} \frac{\sum_{j=1 . . i}\left\lceil\frac{t}{T_{j}}\right\rceil C_{i}}{t} \leq 1$

In essence, the Hyperplanes Exact Test works by reducing the number of points in $S_{i}$ that need to be checked. In [19], Bini and Buttazzo showed that the only points that need to be checked, to determine the schedulability of a task $\tau_{i}$ with $D_{i} \leq T_{i}$, are those in the set $P_{i-1}\left(D_{i}\right)$, where the set of points $P_{j}(t)$ is recursively defined as follows:

$P_{0}(t)=\{t\}$

$P_{j}(t)=P_{j-1}\left(\left\lfloor\frac{t}{T_{j}}\right\rfloor T_{j}\right) \cup P_{j-1}(t)$

The interested reader is referred to [19] for further details of the Hyperplanes Exact Test, including a worked example of its operation.

\section{Performance of RTA and HET schedulability tests}

In [19], Bini and Buttazzo provided evidence showing that the Hyperplanes Exact Test outperforms Response Time Analysis based schedulability tests, starting with the default initial value or the initial values given by Sjodin and Hansson [13] - see figures 6 and 7 in [19] for further details.

We were therefore initially surprised to observe how poorly the HET algorithm performed on our randomly generated tasksets. Figure 20 below shows the frequency distribution of the number of ceiling operations required by the HET algorithm for 10,000 tasksets, each comprising 24 tasks with an overall utilisation of $95 \%$, and a range of task periods spanning 4 orders of magnitude. On average, the HET algorithm required 23,365 ceiling operations, compared with 3,253 ceiling operations for the RTA algorithm, using the default initial value.

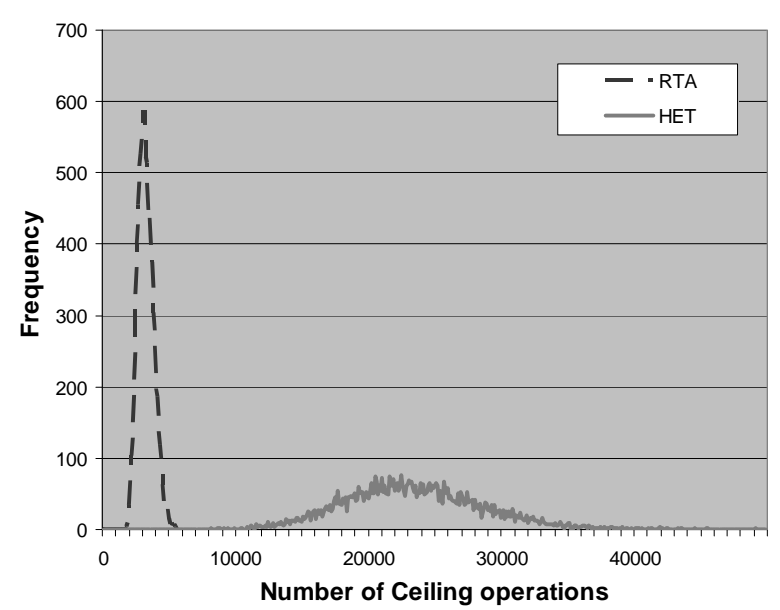

Figure 20: Frequency distribution of the number of "ceiling operations" required by HET and RTA exact schedulability tests, for 


\section{0,000 tasksets with $95 \%$ utilisation.}

Further investigation, as to why these results differ so widely from those reported in [19], revealed that the HET algorithm is extremely sensitive to the distribution of task periods; something that is not immediately apparent from the results presented in [19].

In the experiments reported in [19], task periods were chosen from the range $[1,1,000,000]$ according to a uniform distribution, and the results averaged across $10^{8}$ tasksets. This effectively meant that only tasksets with 1 order of magnitude range of task periods were properly represented in the data. This can be seen by noting that the probability of choosing a task period of less than 10,000 from the range $[1,1,000,000]$ is $1 \%$ when a uniform distribution is used, similarly, the probability of choosing a period of less than 1000 is just $0.1 \%$.

TABLE 5: HET V. RTA ALGORITHMS, NUMBER OF CEILING OPERATIONS

\begin{tabular}{l|llllll}
\hline & \multicolumn{6}{|c}{ Orders of magnitude spanning task periods } \\
Algorithm & $\mathbf{1}$ & $\mathbf{2}$ & $\mathbf{3}$ & $\mathbf{4}$ & $\mathbf{5}$ & $\mathbf{6}$ \\
\hline RTA & 1247 & 1652 & 2050 & 2462 & 2847 & 3297 \\
HET & 380 & 1326 & 5642 & 23014 & 81636 & 197642 \\
\hline HET/RTA & 0.3 & 0.8 & 2.75 & 9.35 & 28.7 & 60.0 \\
\hline
\end{tabular}

Table 5 shows how the average number of ceiling operations required by both the HET algorithm and the RTA algorithm (starting with the default initial value), varied with the number of orders of magnitude spanning task periods, for 10,000 tasksets, each comprising 24 tasks with an overall utilisation of $85 \%$. Here $24 / M$ task periods were chosen according to a uniform distribution from each of the $M$ order of magnitude ranges used (i.e. 100010000 , 10000-100000, 100000-1000000 etc). Note, we used $85 \%$ utilization tasksets as at higher utilizations, too few tasksets were generated that were schedulable with 1 order of magnitude range of task periods.

For tasksets with a range of task periods amounting to 1 order of magnitude, the HET algorithm performs well, outperforming the default RTA approach, at least in terms of the average number of ceiling operations required. This confirms the results published in [19]. However, the execution requirements of the HET algorithm grow exponentially with an increasing range of task periods, so that for the sample tasksets with periods spanning 6 orders of magnitude, the number of ceiling operations required by the HET algorithm is on average 60 times that of the RTA approach. This behaviour with respect to the range of task periods is inherent in the HET algorithm, which in the worst-case can require schedulability to be checked for $2^{n}$ points to determine the schedulability of task $\tau_{n}$.

We note that Figure 20 and Table 5 actually overstate the performance of the HET algorithm. In the HET algorithm, each "ceiling operation" corresponds to a call ${ }^{4}$ of

\footnotetext{
${ }^{4}$ Note, we only counted calls that did not exit via the short circuit returns at the start of the Workload function as "ceiling operations".
}

the recursive WorkLoad() function given in Figure 21 (see [19] for further details of this function, which is the core component of the HET algorithm). The code for the WorkLoad() function is much more complex and takes longer to execute than the code associated with the inner loop of the RTA algorithm, lines 7-10 in Figure 2 (also counted as a "ceiling operation").

To examine the actual execution time of the HET algorithm, we implemented it in $\mathrm{C}$, compiled it using the GNU $\mathrm{C}$ compiler (using the $-\mathrm{O} 2$ option), and ran it on the MPC555 microprocessor used for the execution time measurements reported in Section 7. To obtain the best possible performance from the HET algorithm, we made some simple improvements to the algorithm as presented in [19]; avoiding the use of floating point arithmetic, and instead using 32-bit integers for task parameters and computed values. The basic code for the Workload function is given in Figure 21. Note that the ceiling() and $\min ()$ functions were implemented as Macros, so as to avoid function call overheads. We subsequently also removed the short circuit returns from the original implementation, instead coding the algorithm so that the final 'leaf' calls to the Workload function were not required. This reduced the overall number of calls to the Workload function by just over a factor of 2 , significantly reducing the overall execution time.

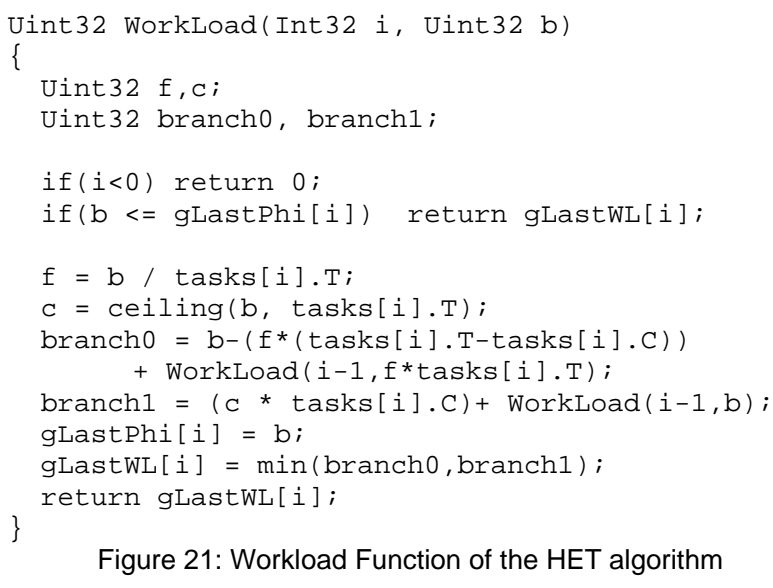

Execution time measurements were taken using the RapiTime worst-case execution time analysis toolset [24].

Applied to the same 95\% utilisation taskset referred to in Section 7, the HET algorithm required 19181 ceiling operations to determine that the taskset was schedulable, corresponding to 3,196,748 clock cycles, some 19 times longer than the RTA algorithm using the default initial value, and some 144 times longer than the most efficient approach (\#9) reported in Section 7.

The average time for each "ceiling operation" in the HET algorithm, (effectively corresponding to a call to the Workload function) was 167 clock cycles (down from 228 clock cycles with the short circuit returns present); by comparison, the RTA algorithm required on average just 26 clock cycles for each iteration of its inner loop (i.e. 26 
clock cycles per "ceiling operation"). From this data, we infer that the apparent performance advantage of the HET algorithm for small ranges of task periods would appear to be illusionary. Correcting the figures from Table 5 to account for the differences in execution times for each iteration of the two algorithms, results in the data presented in Table 6 and Figure 22.

TABLE 6: HET V. RTA ALGORITHMS, EXECUTION TIME IN CLOCK CYCLES X 1000

\begin{tabular}{l|llllll}
\hline & \multicolumn{6}{|c}{ Orders of magnitude spanning task periods } \\
Algorithm & $\mathbf{1}$ & $\mathbf{2}$ & $\mathbf{3}$ & $\mathbf{4}$ & $\mathbf{5}$ & $\mathbf{6}$ \\
\hline RTA & 32.4 & 43.0 & 53.3 & 64.0 & 74.0 & 85.7 \\
HET & 63.1 & 221.5 & 942 & 3843 & 13633 & 33006 \\
\hline HET/RTA & 1.95 & 5.2 & 17.7 & 60 & 184 & 385 \\
\hline
\end{tabular}

The final row in Table 6 records the factor by which the execution time of the HET algorithm exceeds that of the RTA algorithm. We infer from this data, that in practice, the RTA algorithm generally outperforms the HET algorithm, and by some significant margin in the case of tasksets with a broad spread of task periods.

It is still possible that the HET algorithm may outperform the RTA algorithm for some tasksets comprising small numbers of tasks (so the number of scheduling points inspected by the HET algorithm is small), and with a small range of task periods. However, we argue that improving upon the RTA algorithm under these conditions is of little practical value as the RTA test has a sufficiently short execution time in this domain (low number of tasks, small spread of task periods) anyway.

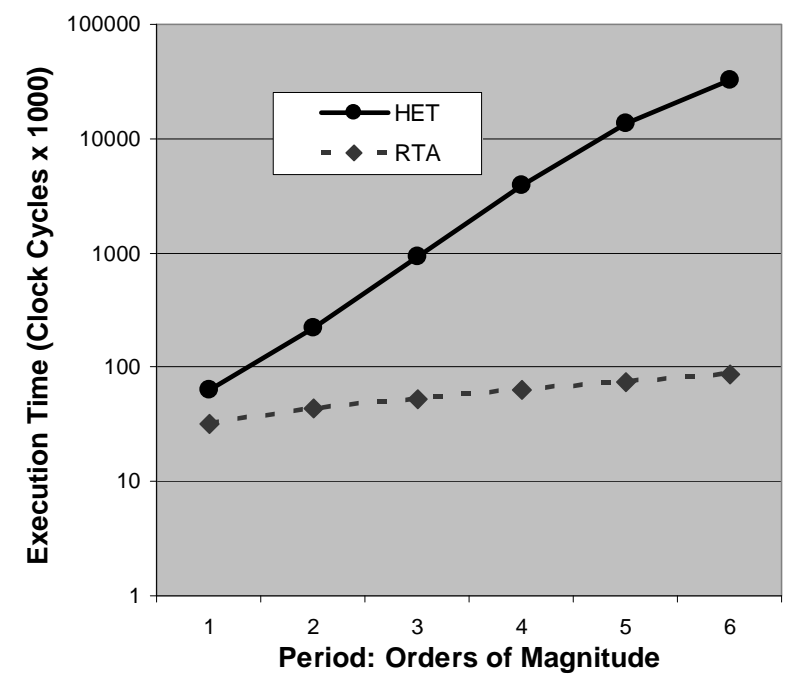

Figure 22: Average execution time required by the HET and RTA exact schedulability tests, plotted against the number of orders of magnitude range of task periods, for tasksets with $85 \%$ utilisation.

\section{Acknowledgements}

This work was funded in part by the EU Frescor project.

\section{References}

[1] M.S. Fineberg and O. Serlin, "Multiprogramming for Hybrid Computation". In proceedings AFIPS Fall Joint Computing Conference, pp. 1-13, 1967

[2] O. Serlin, "Scheduling of Time Critical Processes". In proceedings AFIPS Spring Computing Conference, pp 925-932, 1972.

[3] C.L. Liu and J.W. Layland. "Scheduling Algorithms for Multiprogramming in a Hard-Real-time Environment", Journal of the ACM, 20(1): 46-61, January 1973.

[4] J.Y.-T. Leung and J. Whitehead, "On the Complexity of Fixed-Priority Scheduling of Periodic Real-time Tasks". Performance Evaluation, 2(4): 237-250, December 1982.

[5] M. Joseph and P.K. Pandya. "Finding Response Times in a Real-time System”. The Computer Journal, 29(5):390-395, October 1986.

[6] J. P. Lehoczky, L. Sha, and Y. Ding, "The Rate-Monotonic Scheduling Algorithm: Exact Characterization and Average Case Behavior". In proceedings of the 10th IEEE Real-Time Systems Symposium, pp. 166-172, 1989.

[7] L. Sha, R. Rajkumar, and J.P. Lehoczky. "Priority Inheritance Protocols: An Approach to Real-time Synchronization”. IEEE Transactions on Computers, 39(9): 1175-1185, 1990.

[8] J.P. Lehoczky. "Fixed Priority Scheduling of Periodic Task Sets with Arbitrary Deadlines". In Proceedings 11th IEEE RealTime Systems Symposium, pp. 201-209, IEEE Computer Society Press, December 1990.

[9] T.P. Baker. "Stack-based Scheduling of Real-Time Processes.” Real-Time Systems Journal (3)1, pp. 67-100, 1991.

[10] K.W. Tindell. "Using Offset Information to Analyse Static Priority Pre-Emptively Scheduled Task Sets". Technical Report YCS-92-182. Dept. of Computer Science, University of York, UK, 1992.

[11] N.C. Audsley, A. Burns, M. Richardson, K.W. Tindell, and A.J. Wellings. "Applying New Scheduling Theory to Static Priority Pre-emptive Scheduling". Software Engineering Journal, 8(5):284-292, September 1993.

[12] K.W. Tindell, A. Burns, A.J. Wellings. "An Extendible Approach for Analyzing Fixed Priority Hard Real-time Tasks". Real-Time Systems. Volume 6, Number 2, pp133-151 March 1994.

[13] M. Sjodin and H. Hansson. "Improved Response Time Analysis Calculations". In Proceedings of the 19th IEEE RealTime Systems Symposium, pages 399-408, Madrid, Spain, December 1998.

[14] R.J. Bril, W.F.J. Verhaegh, and E-J.D. Pol. "Initial Values for On-line Response Time Calculations". In Proceedings of the 15th Euromicro Conference on Real-Time Systems, pages 13-22, Porto, Portugal, July 2003.

[15] E. Bini and G.C. Buttazzo. "Measuring the Performance of Schedulability tests". Real-Time Systems, 30(1-2):129-154,May 2005.

[16] W-C. Lu, J-W. Hsieh, W-K. Shih, T-W. Kuo. "A Faster Exact Schedulability Analysis for Fixed-priority Scheduling". Journal of Systems and Software, Vol. 79, Issue 12, pp. 1744- 
1753, December 2006.

[17] W-C. Lu, K-J. Lin, H-W. Wei1, and W-K. Shih. "PeriodDependent Initial Values for Exact Schedulability Test of Rate Monotonic Systems". In Proceedings Parallel and Distributed Processing Symposium 2007, IPDPS 2007. Long Beach, USA, pp. 1-8, March 2007.

[18] E. Bini and S.K. Baruah. "Efficient Computation of Response Time Bounds under Fixed-priority Scheduling”. In Proceedings of the 15th conference on Real-Time and Network Systems, pp. 95-104, Nancy, France, March 2007.

[19] E. Bini and G.C. Buttazzo. "Schedulability Analysis of Periodic Fixed Priority Systems". IEEE Transactions on Computers, 53(11):1462-1473, November 2004.

[20] A. Zuhily and A. Burns "Optimality of (D-J)-monotonic Priority Assignment”. Information Processing Letters. Number 103, pp. 247-250, April 2007.

[21] R.J. Bril, J.J. Lukkien, W.F.J. Verhaegh. "Worst-Case Response Time Analysis of Real-Time Tasks under Fixed-Priority Scheduling with Deferred Preemption Revisited". In Proceedings of the 19th Euromicro Conference on Real-Time Systems ECRTS. pp. 269-279. July 2007.

[22] N.C. Audsley, "Flexible Scheduling of Hard Real-Time Systems". PhD Thesis. Dept. of Computer Science, University of York, UK, 1993.

[23] http://www.frescor.org/ 2007.

[24] "RapiTime White Paper: Worst-case Execution Time Analysis" available from www.rapitasystems.com 2007.

[25] R.I. Davis, A. Burns, R.J. Bril, and J.J. Lukkien. "Controller Area Network (CAN) Schedulability Analysis: Refuted, Revisited and Revised". Real-Time Systems, Volume 35, Number 3, pp 239-272. April 2007.

[26] E. Bini, G.C. Buttazzo, and G.M. Buttazzo. "Rate Monotonic Scheduling: The Hyperbolic Bound". IEEE Transactions on Computers, 52(7):933-942, July 2003.

[27] S. Lauzac, R. Melhem, and D. Mosse. "An Improved Ratemonotonic Admission Control and its Applications". IEEE Transactions on Computers, 52(3):337-350, March 2003. 